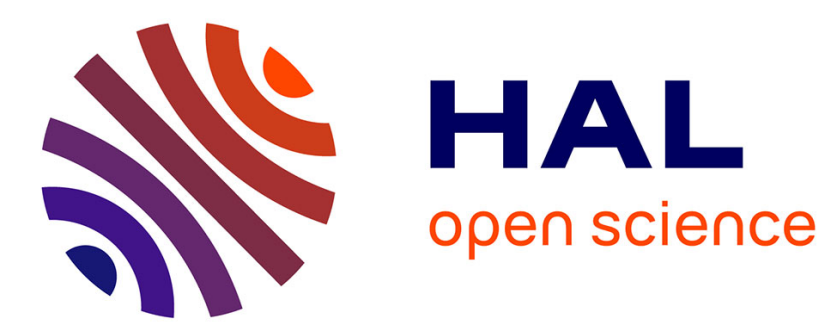

\title{
Predictor-feedback prescribed-time stabilization of LTI systems with input delay
}

\author{
Nicolás Espitia, Wilfrid Perruquetti
}

\section{To cite this version:}

Nicolás Espitia, Wilfrid Perruquetti. Predictor-feedback prescribed-time stabilization of LTI systems with input delay. IEEE Transactions on Automatic Control, In press, 10.1109/TAC.2021.3093527 . hal-03275335

\section{HAL Id: hal-03275335 \\ https://hal.science/hal-03275335}

Submitted on 1 Jul 2021

HAL is a multi-disciplinary open access archive for the deposit and dissemination of scientific research documents, whether they are published or not. The documents may come from teaching and research institutions in France or abroad, or from public or private research centers.
L'archive ouverte pluridisciplinaire HAL, est destinée au dépôt et à la diffusion de documents scientifiques de niveau recherche, publiés ou non, émanant des établissements d'enseignement et de recherche français ou étrangers, des laboratoires publics ou privés. 


\title{
Predictor-feedback prescribed-time stabilization of LTI systems with input delay
}

\author{
Nicolás Espitia and Wilfrid Perruquetti
}

\begin{abstract}
This paper deals first with the problem of prescribed-time stability of linear systems without delay. The analysis and design involve the Bell polynomials, the generalized Laguerre polynomials, the Lah numbers and a suitable polynomialbased Vandermonde matrix. The results can be used to design a new controller -with time-varying gains- ensuring prescribedtime stabilization of controllable LTI systems. The approach leads to similar results compared to Holloway et al. 2019 but offers an alternative and compact control design (specially for the choice of the time-varying gains). Based on the preliminary results for the delay-free case, we then study controllable LTI systems with single input and subject to a constant input delay. We design a predictor feedback with time-varying gains. To achieve this, we model the input delay as a transport PDE and build on the cascade PDE-ODE setting (inspired by Krstic. 2009) so as the design of the prescribed-time predictor feedback is carried out based on the backstepping approach which makes use of timevarying kernels. We guarantee the bounded invertibility of the backstepping transformation and we prove that the closed-loop solution converges to the equilibrium in a prescribed-time. A simulation example illustrates the results.
\end{abstract}

Index Terms-Infinite dimensional systems, delay systems, prescribed-time convergence, backstepping control design.

\section{INTRODUCTION}

$\mathbf{F}$ Inite and fixed-time stabilization and estimation have been extensively considered in the framework of linear and nonlinear ordinary differential equations (ODEs) (see e.g. [18], [6], [35], [29], [21] and references therein). The need to meet some performance, time constraints and precision has highly motivated the stabilization and estimation in finitetime/fixed-time. More recently, prescribed-time concepts have arisen to achieve a more demanding type of convergence: the time of convergence can be prescribed in the design independently of initial conditions [37], [20], [26], [40], [42]. This type of convergence has emerged for tactical and strategic missile guidance problems but it is not limited to them. Many numerous applications (e.g. rendezvous, spacecraft docking, trajectory tracking for nonholonomic mobile robots, finite-time deployment and formation control for multi-agent systems, weather forecasting) require the transient process must occur within a given time.

For infinite dimensional systems, namely partial differential equations (PDEs), finite, fixed and prescribed-time concepts have also become an attractive research area as PDEs may indeed describe many complex systems (e.g. hydraulic networks, tubular chemical reactors, etc). Meeting time constraints or just

*This work has been partially supported by ANR Project Finite4SoS (ANR 15-CE23-0007). N. Espitia and W. Perruquetti are with Univ. Lille, CNRS, Centrale Lille, UMR 9189 CRIStAL, F-59000 Lille, France. Corresponding author: nicolas.espitia-hoyosduniv-lille.fr realizing the well-known separation principle are central issues that can be achieved by means of finite-/fixed- and prescribedtime issues. On one hand, for hyperbolic PDE systems, some contributions on stabilization in finite-time can be highlighted: see e.g. [33], [11], [2], [11], [13]. On the other hand, for linear parabolic PDEs, some works have addressed some relevant issues on null controllability and finite-time stabilization (e.g. [12]) and prescribed-time stabilization [16], [38] which make uses of the backstepping approach with time-varying kernels. In the context of infinite dimensional systems in abstract formulation, one can refer [34] which exploits homogeneity arguments.

In the framework of time-delay systems, it is known that most of the results on stabilization and estimation are based on asymptotic or exponential guarantees. In particular, exponential stabilization of LTI systems with input delay can be performed based on the predictor feedback. In [28], [27], under a PDE-ODE cascade setting, the classical predictor is brought back by making use of the backstepping approach. One of the advantages of this approach is that one can perform an easier Lyapunov-based stability analysis on the so-called target system which is chosen with the desired stability properties. The backstepping PDE framework for time-delay systems has been extended to deal with delay adaptive control, nonlinear systems with input delay, time-varying delay and distributed input delay [8], [7], [3] to mention a few. Other techniques relying on model reduction for linear time-varying systems with delays can be found in e.g. [30]. However, finite-/fixed/prescribed-time concepts for time-delay systems have not achieved a sufficient level of maturity and still constitute a challenging topic. One may refer to some of the pioneering contributions for time-delay systems e.g. [25] and [31], the latter having pointed out some key obstructions for the design of static finite-time controllers (see also [14]) and which came up with a controller based on Artstein's transformation [1] to stabilize in finite-time with a settling time depending on initial conditions of the system. Besides, by building upon such a transformation, and using some homogeneous controllers, fixed-time stabilization is achieved for general LTI systems with input delay as in [44]. Although, to the best of our knowledge, prescribed-time stabilization for time-delay systems and for LTI systems with input delays has not been studied yet in the literature.

In this work, we address the problem of prescribed-time stabilization of controllable LTI systems with input delay. We rely on a PDE-ODE cascade setting and on the use of an invertible Volterra backstepping transformation whose kernels are time-varying. By combining the ideas of [20] and [16], 
we propose a target system which is prescribed-time stable. Then, we relate back such a property to the original system through a suitable characterization of the bounded invertibility of the backstepping transformation. For the stability analysis and control design, compact formulations using Bell polynomials, generalized Laguerre polynomials, elementary symmetric polynomials and polynomial-based Vandermonde matrix are studied. The resulting boundary controller resembles very much to the classical predictor. In this framework, it contains time-varying functions as the kernel turns out to be time varying. We call it prescribed-time predictor control. A conference paper [15] contains some preliminary results on prescribed-time predictor control for a scalar linear equation with input delay and includes also a brief overview of some results for the $n$-dimensional case that are fully covered in this paper. Indeed, in contrast to the conference version, this paper deals with $n$-dimensional linear systems with single delayed input and provides the complete results and details for all proofs as well as a detailed analysis and discussions on the novel prescribed-time predictor control and the bounded invertibility of the the backstepping transformation with timevarying kernels.

This paper is organized as follows. In Section II, we introduce some preliminaries on prescribed-time stability of linear systems without delay. In Section [II], we consider controllable LTI systems with input delay. We use a PDEODE setting under a backstepping approach, we discuss the bounded invertibility of the transformation and we derive the prescribed-time predictor control. In Section $[\mathrm{V}$ we consider a numerical example to illustrate the main results. Finally, conclusions and perspectives are given in Section VI Some technical proofs are given in the Appendix.

Notations: $\mathbb{R}_{+}$denotes the set of nonnegative real numbers. For non zero integers $m$ and $n$, let $0_{m \times n}$ be the $(m, n)$-matrix with zero entries, $I_{m}$ be the identity matrix of dimension $m, J_{n}=\left(\left(0_{(n-1) \times 1}, I_{n-1}\right)^{\top}, 0_{n \times 1}\right)^{\top}$ (Jordan matrix) and $\mathcal{L}_{n}(s)=\left(0_{(n) \times(n-1)}, s\right)^{\top}$, where $s \in \mathbb{R}^{n}$. Let $1 \leq p \leq \infty$, then the induced norm of a $(n, n)-$ matrix $M$ is defined as $\|M\|_{p}=\sup \left\{\|M x\|_{p}: x \in \mathbb{R}^{n}\right.$ with $\left.\|x\|_{p}=1\right\}$ where $\|x\|_{p}$ is the $p$-vector norm of $x$. Thus, when $p=1$ (respectively $p=\infty$ ), $\|M\|_{p}$ corresponds respectively to the maximum absolute row (resp. column) sum norm. We recall that $\|M\|_{2} \leq \sqrt{\|M\|_{1}\|M\|_{\infty}} \cdot L_{m}^{(\alpha)}(\cdot)$ denotes the generalized Laguerre polynomial. $\left(\begin{array}{l}n \\ k\end{array}\right):=\frac{n !}{k !(n-k) !}, k=1,2, . ., n$ denotes the binomial coefficients. $B_{n}(\cdot)$ denotes the complete exponential Bell polynomials and $\sigma_{n}(\cdot)$ denotes the elementary symmetric polynomials. The set of all functions $g:[0, h] \rightarrow$ $\mathbb{R}^{n}$ such that $\int_{0}^{h} g(x)^{2} d x<\infty$ is denoted by $L^{2}\left((0, h), \mathbb{R}^{n}\right)$. A continuous function $\alpha:[0, a) \subset \mathbb{R}_{+} \rightarrow \mathbb{R}_{+}, r \mapsto \alpha(r)$, is said to be a class- $\mathcal{K}$ function if it is strictly increasing with $\alpha(0)=0 . \alpha$ is a class- $\mathcal{K}_{\infty}$ function if it is a class- $\mathcal{K}$ function with $a=\infty$ and $\alpha(r) \rightarrow \infty$ as $r \rightarrow \infty$. A continuous function $\beta:[0, a) \subset \mathbb{R}_{+} \times \mathbb{R} \rightarrow \mathbb{R}_{+},(r, t) \mapsto \beta(r, t)$, belongs to class- $\mathcal{K} \mathcal{L}$ if for each fixed $t$, the mapping $r \mapsto \beta(r, t)$ belongs to class $\mathcal{K}_{\infty}$ with respect to $r$; and, for each fixed $r \in \mathbb{R}_{+}$, the mapping $t \mapsto \beta(r, t)$ is decreasing with respect to $t$ and $\lim _{t \rightarrow+\infty} \beta(t, r)=0$.

\section{PRELIMINARIES ON FIXED-TIME STABILITY IN PRESCRIBED-TIME}

Before considering the control design for linear systems with input delay (whose problem formulation is stated in Section [II), we are going to deal first with some relevant preliminary results on fixed-time stability in prescribed-time for linear systems without delay. These preliminaries will allow us to prepare the field and have compact formulations that will simplify the control design analysis for delayed case which is studied in Section III

Let us first recall the following definition of "fixed-time globally uniformly asymptotically stable" (in short FT-GUAS) adopted from [20]:

Definition 1: The origin of the system $\dot{z}=f(t, z)$ is said to be $F T-G U A S$ if there exist a class $\mathcal{K} \mathcal{L}$ function $\beta$ and a function $\mu:\left[t_{0}, t_{0}+T\right) \rightarrow \mathbb{R}_{+}$such that $\mu$ tends to infinity as $t$ goes to $t_{0}+T$ and, $\forall t \in\left[t_{0}, t_{0}+T\right)$

$$
\|z(t)\| \leq \beta\left(\left\|z\left(t_{0}\right)\right\|, \mu\left(t-t_{0}\right)\right),
$$

where $T$ is a time that can be prescribed in the design.

\section{A. Scalar Time-varying linear autonomous system}

Let us briefly study the previous stability property on a scalar time-varying linear autonomous system without delay. The analysis will be then extended to a general case.

The next lemma gives a sufficient condition for FT-GUAS of the following scalar system:

$$
\dot{z}(t)=-c(t) z(t), \quad z\left(t_{0}\right)=z_{0} .
$$

Lemma 1: Let $c \in L_{\text {loc }}^{1}\left[t_{0}, t_{0}+T\right.$ ) with $c(t)>0$ (a.e on $\mathbb{R}$ ), then (1) is FT-GUAS with a prescribed time $T>0$ if $\lim _{t \rightarrow t_{0}+T} \hat{c}(t)=+\infty$ where $\hat{c}(t)=\int_{t_{0}}^{t} c(\tau) d \tau$.

Proof: Without loss of generality, we set $t_{0}=0$ (the proof remains the same otherwise). Let us note that as long as $c$ is integrable, a direct integration of (1) gives $z(t)=$ $\exp (-\hat{c}(t)) z(0)$ thus $|z(t)|=\left|z_{0}\right| \exp (-\hat{c}(t))$. As $c(t)>0$, a.e, then $\hat{c}$ is increasing on $[0, T]$, thus its inverse function $\hat{c}^{-1}$ exists. Let $\tau(s)=\frac{T}{\hat{c}^{-1}(s)}-1$, then $\tau(\hat{c}(t))=\frac{T}{t}-1$ which is a $\mathcal{L}_{\infty}$ function on $(0, T)$. Now defining $\beta(r, t)=r \exp (-\alpha(t-$ 1)) where $\alpha(s)=\left(\frac{1}{\tau(s)}\right)^{-1}$ (the inverse function of $\frac{1}{\tau(s)}$ clearly exists). Since we have $\alpha(0)=0$, then $\alpha \in \mathcal{K}_{\infty}$. Note that, $\hat{c}(t)=\alpha\left(\frac{t}{T-t}\right)$, thus it yields: $|z(t)|=\beta(|z(0)|, \mu(t))$ and $\beta$ being of class $-\mathcal{K} \mathcal{L}$.

Remark 1: There exist many functions meeting the sufficient condition for FT-GUAS in Lemma 1 As a matter of example, one can consider blow-up functions having the form $c(t)=$ $\frac{T^{\epsilon}}{\left.T+t_{0}-t\right)^{\epsilon}}$ where $\epsilon>1$ as in [37], [20].

In this paper, we will use the following blow-up function (borrowed from [16]) that will be useful for having a more compact formulation for the stability analysis. We consider

$$
c(t)=\frac{\bar{c}_{0}^{2} T^{2}}{\left(T+t_{0}-t\right)^{2}}, \quad c\left(t_{0}\right)=\bar{c}_{0}^{2},
$$

with $\bar{c}_{0}, T>0$. Thus, (1) is FT-GUAS in light of Lemma 1 


\section{B. Linear time-varying systems in companion canonical form}

Without loss of generality, we consider a time-varying system given in a companion canonical form.

$$
\dot{z}(t)=C(t) z(t), \quad z \in \mathbb{R}^{n},
$$

where $C(t)=J_{n}+\mathcal{L}_{n}(-p(t))$, i.e.,

$$
C(t)=\left(\begin{array}{ccccc}
0 & 1 & 0 & \cdots & 0 \\
0 & 0 & 1 & \cdots & 0 \\
\vdots & \vdots & \vdots & \ddots & \vdots \\
0 & 0 & 0 & \cdots & 1 \\
-p_{0}(t) & -p_{1}(t) & -p_{2}(t) & \cdots & -p_{n-1}(t)
\end{array}\right)
$$

with $p(t)=\left(p_{0}(t), \ldots, p_{n-1}(t)\right)$, that will be characterized later on. It is worth recalling that the unique solution to (3) is given by $z(t)=\Phi_{C}\left(t, t_{0}\right) z\left(t_{0}\right)$ where $\Phi_{C}$ is the statetransition matrix satisfying $\frac{\partial}{\partial t} \Phi_{C}\left(t, t_{0}\right)=C(t) \Phi_{C}\left(t, t_{0}\right)$ and $\Phi_{C}(t, t)=I_{n}$. Moreover, there exists a coordinate transformation [23],

$$
\bar{z}(t)=V^{-1}(t) z(t),
$$

which converts (3) into the following system:

$$
\dot{\bar{z}}(t)=D(t) \bar{z}(t)
$$

where $D$ is diagonal matrix such that $V$ satisfies the following relation from [24], [43]:

$$
V(t) D(t)=C(t) V(t)-\dot{V}(t) .
$$

Indeed, consider

$$
V(t)=\left(\begin{array}{ccc}
1 & \cdots & 1 \\
\left(\delta^{0}\left(-r_{1} c\right)\right)(t) & \cdots & \left(\delta^{0}\left(-r_{n} c\right)\right)(t) \\
\vdots & \ddots & \vdots \\
\left(\delta^{n-2}\left(-r_{1} c\right)\right)(t) & \cdots & \left(\delta^{n-2}\left(-r_{n} c\right)\right)(t)
\end{array}\right),
$$

which is a Generalized Vandermonde matrix where the operator $\delta$ is defined recursively by:

$$
\begin{aligned}
\left(\delta^{0}\left(-r_{i} c\right)\right)(t)= & -r_{i} c(t), \\
\left(\delta^{n}\left(-r_{i} c\right)\right)(t)= & \left(\delta\left(\delta^{n-1}\left(-r_{i} c\right)\right)\right)(t) \\
= & \left(-r_{i} c\right)(t)\left(\delta^{n-1}\left(-r_{i} c\right)\right)(t) \\
& +\frac{d}{d t}\left(\delta^{n-1}\left(-r_{i} c\right)\right)(t) .
\end{aligned}
$$

where the function $c(t)$ is defined in (2), and where the coefficients $r_{i}, i=1, \ldots, n$ are different positive real numbers $\left(r_{i}>0, r_{i} \neq r_{j}\right.$ for $i \neq j$ in the range $\left.1, \ldots n\right)$. Hence, $D(t)=\operatorname{diag}\left(-r_{1} c(t),-r_{2} c(t), \cdots,-r_{n} c(t)\right)$.

We can further provide characterizations of such a transformation that will be instrumental for the prescribed-time stability property of (3); and more importantly, when dealing with input delayed case. Those characterizations are given in terms of special functions such as Bell polynomials, generalized Laguerre polynomials ${ }^{1}$, the elementary symmetric polynomials and the Lah numbers (see Appendix $\mathrm{A}$ for more details about the special functions used herein).

\footnotetext{
${ }^{1}$ see [17], [16] for related studies but in the context of reaction-diffusion PDEs.
}

Proposition 1: Let $V(t)$ be given by (8) with operator $\delta$ defined by (9). Then, the following holds true:

$$
\left(\delta^{n}\left(-r_{i} c\right)\right)(t)=B_{n+1}\left(-r_{i} c(t), \ldots,-r_{i} c^{(n)}(t)\right) .
$$

where $B_{n+1}(\cdot)$ denotes the complete exponential Bell polynomials.

Proof: It is an immediate consequence of the application of the Faà di Bruno formula in terms of Bell polynomials.

Proposition 2: Let $V(t)$ be given by (8) with operator $\left(\delta^{n}\left(-r_{i} c\right)\right)(t)$ satisfying (10) and let $D(t)$ and $C(t)$ satisfy (7). Then, we have the relation

$$
\left(\delta^{n}\left(-r_{i} c\right)\right)(t)=\frac{-r_{i} c(t)(\sqrt{c(t)})^{n}}{\left(\bar{c}_{0} T\right)^{n}} n ! L_{n}^{(1)}\left(r_{i} \bar{c}_{0} T \sqrt{c(t)}\right) .
$$

Proof: See Appendix $\mathrm{B}$

The above formula (11) suggests to introduce the following powered "square" function for some $r \in \mathbb{R}, v \in \mathbb{Z}$ :

$$
\mathfrak{s}_{r}^{v}(t)=r c(t)\left(\frac{\sqrt{c(t)}}{\bar{c}_{0} T}\right)^{v},
$$

which will simplify the notation. For example (11) succinctly reads as $\left(\delta^{n}\left(-r_{i} c\right)\right)(t)=n ! \mathfrak{s}_{-r_{i}}^{n}(t) L_{n}^{(1)}\left(\mathfrak{s}_{r_{i}}^{-1}(t)\right)$.

Notice that $V(t)$ (8) is made up of terms involving the generalized Laguerre polynomials, yielding a special polynomialbased Vandermonde matrix, thanks to which we have the following result.

Proposition 3: Under the assumptions of Proposition 2, the components $p_{0}(t), p_{j}(t)(j=1, \ldots n-1)$ of $C(t)$ (4) are explicitly given by the following formulas:

$$
p_{0}(t)=\sigma_{n}\left(r_{1}, . ., r_{n}\right) c^{n}(t),
$$

and for $j=1, \ldots, n-1$,

$$
\begin{aligned}
p_{j}(t)= & \frac{(\sqrt{c(t)})^{n-j}}{\left(\bar{c}_{0} T\right)^{n-j}} \sum_{k=j}^{n}(-1)^{k-j} \sigma_{n-k}\left(r_{1}, \ldots, r_{n}\right) \\
& \times \operatorname{Lah}(k, j)\left(\bar{c}_{0} T \sqrt{c(t)}\right)^{n-k},
\end{aligned}
$$

where $\sigma_{n-k}(\cdot)$ are the elementary symmetric polynomials given by (102)-(104) (see Appendix A and $\operatorname{Lah}(k, j)$ are the Lah numbers given by Property 2 in Appendix $\mathrm{A}$.

\section{Proof: See Appendix C.}

\section{FT-GUAS characterization for (3)}

Let us introduce the following "Exponential"-like function

$$
\mathfrak{\ell}_{r}(t)=\exp \left(\mathfrak{s}_{r}^{-1}(t)\right)=\exp \left(r \bar{c}_{0} T \sqrt{c(t)}\right)
$$

for some $r \in \mathbb{R}$, where $\bar{c}_{0}, T$ and $c(t)$ are given in (2). In addition, we introduce the "Diagonal" matrix

$$
\mathfrak{D}(t)=\operatorname{diag}\left(\mathfrak{e}_{-r_{1}}(t), \ldots, \mathfrak{e}_{-r_{n}}(t)\right) .
$$

We state next the FT-GUAS property for (3).

Lemma 2: Let $C(t)=J_{n}+\mathcal{L}_{n}(-p(t))$ given by (4) with $p$ satisfying (13)-(14) and the function $c(t)$ is defined by (2). Let $D(t)=\operatorname{diag}\left(-r_{1} c(t),-r_{2} c(t), \cdots,-r_{n} c(t)\right)$ satisfying (7) where $V(t)$ is defined by (8) with $\left(\delta^{n}\left(-r_{i} c\right)\right)(t)$ given by 
(11) as in Proposition 2. Then, for any $z_{0} \in \mathbb{R}^{n}$, the solution to system (3) is FT-GUAS, i.e. there exists $\eta_{z}>0$ and a positive polynomial $P$ in $\sqrt{c(t)}$ such that

$$
\|z(t)\|^{2} \leq \eta_{z} P(\sqrt{c(t)}) \mathfrak{e}_{-r_{\min }}(t)\left\|z\left(t_{0}\right)\right\|^{2},
$$

for all $t \in\left[t_{0}, t_{0}+T\right)$, where $r_{\text {min }}=\min _{i=1, \ldots, n}\left\{r_{i}\right\}$. In particular, it holds

$$
\|z(t)\| \rightarrow 0, \quad t \rightarrow t_{0}+T .
$$

Proof: For $t \in\left[t_{0}, t_{0}+T\right)$, matrices $C(t), V(t)$, $V^{-1}(t), D(t)$ exist and are well defined. Thus, by using (5), system (3) is transformed into $\dot{\bar{z}}(t)=D(t) \bar{z}(t)$, where $D(t)=\operatorname{diag}\left(-r_{1} c(t), \cdots,-r_{n} c(t)\right)$ such that (7) is satisfied. Therefore, the solution is given as follows:

$$
\bar{z}(t)=\bar{\Phi}_{D}\left(t, t_{0}\right) \bar{z}\left(t_{0}\right), \quad \text { for all } t \in\left[t_{0}, t_{0}+T\right),
$$

where $\bar{\Phi}_{D}\left(t, t_{0}\right)=\exp \left(\int_{t_{0}}^{t} D(s) d s\right)$ is the state transition matrix of the underlying linear (diagonal-form) time-varying system ( $\bar{z}$-dynamics). Moreover, it follows that $\bar{\Phi}_{D}\left(t, t_{0}\right)=$ $\mathfrak{D}(t) \mathfrak{D}^{-1}\left(t_{0}\right)$. Then, from (5), (18), the solution to (3) is given, for all $t \in\left[t_{0}, t_{0}+T\right)$, as follows:

$$
z(t)=\Phi_{C}\left(t, t_{0}\right) z\left(t_{0}\right),
$$

where $\Phi_{C}\left(t, t_{0}\right)$, the state transition matrix of the linear timevarying system ( $z$-dynamics), is

$$
\begin{aligned}
\Phi_{C}\left(t, t_{0}\right) & =\mathfrak{V}(t) \mathfrak{V}^{-1}\left(t_{0}\right), \\
\mathfrak{V}(t) & =V(t) \mathfrak{D}(t),
\end{aligned}
$$

where $\mathfrak{V}(t)$ is a Vandermonde-like matrix. We are interested in the time-evolution of $\left\|\Phi_{C}\left(t, t_{0}\right)\right\|_{2}$, for all $t \in\left[t_{0}, t_{0}+T\right)$. Using (11), (15) and (16), the entries of $\mathfrak{V}(t)$ are $\mathfrak{V}_{1 j}(t)=$ $\mathfrak{e}_{-r_{j}}(t)$ and for $i>1$ :

$$
\mathfrak{V}_{i j}(t)=(i-2) ! \mathfrak{R}_{-r_{j}}(t) \mathfrak{s}_{-r_{j}}^{i-2}(t) L_{i-2}^{(1)}\left(\mathfrak{s}_{r_{j}}^{-1}(t)\right) .
$$

Using a well-established upper bound of the generalized Laguerre polynomials $\left|L_{n}^{(\alpha)}(s)\right| \leq\left(\begin{array}{c}n+\alpha \\ n\end{array}\right) \exp \left(\frac{s}{2}\right)$ [39], we get for $i>1$ :

$$
\left|\mathfrak{V}_{i j}(t)\right| \leq(i-1) ! \mathfrak{R}_{\frac{-r_{j}}{2}}(t) \mathfrak{s}_{r_{j}}^{i-2}(t) .
$$

On one hand, using $\|\mathfrak{V}(t)\|_{2} \leq \sqrt{\|\mathfrak{V}(t)\|_{1}\|\mathfrak{V}(t)\|_{\infty}}$, the following estimate holds, for all $t \in\left[t_{0}, t_{0}+T\right)$ :

$$
\begin{aligned}
\|\mathfrak{V}(t)\|_{2}^{2} & \leq\|\mathfrak{V}(t)\|_{1}\|\mathfrak{V}(t)\|_{\infty} \\
& \leq P(\sqrt{c(t)}) \mathfrak{e}_{-r_{\min }}(t),
\end{aligned}
$$

where $P(\sqrt{c(t)})$ is a polynomial function of $\sqrt{c(t)}$ which can be obtained by computing $\|\mathfrak{V}(t)\|_{1}$ and $\|\mathfrak{V}(t)\|_{\infty}$ using (23). On the other hand, $\left\|\mathfrak{V}^{-1}\left(t_{0}\right)\right\|_{2}^{2} \leq$ $\left\|\mathfrak{V}^{-1}\left(t_{0}\right)\right\|_{1}\left\|\mathfrak{V}^{-1}\left(t_{0}\right)\right\|_{\infty}, \leq \eta_{z}$ where $\eta_{z}$ is some positive constant. Therefore, we obtain, for all $t \in\left[t_{0}, t_{0}+T\right)$

$$
\left\|\Phi_{C}\left(t, t_{0}\right)\right\|^{2} \leq \eta_{z} P(\sqrt{c(t)}) \mathfrak{e}_{-r_{\min }}(t)
$$

thus,

$$
\|z(t)\|^{2} \leq \eta_{z} P(\sqrt{c(t)}) \mathfrak{e}_{-r_{\min }}(t)\left\|z\left(t_{0}\right)\right\|^{2} .
$$

Notice that $P(\sqrt{c(t)})$ blows up as $t$ goes to $t_{0}+T$; nevertheless, the exponential term $\mathfrak{e}_{-r_{\min }}(t)=\exp \left(-r_{\min } \bar{c}_{0} T \sqrt{c(t)}\right)$ decreases faster than the growth-in-time of $P(\sqrt{c(t)})$. Therefore, we can conclude that $\|z(t)\|^{2} \rightarrow 0, \quad$ as $t \rightarrow t_{0}+T$ with exponential soft landing ${ }^{2}$. This concludes the proof.

A direct consequence of Lemma 2 in conjunction with Proposition 3 is the following corollary.

Corollary 1: The following chain of integrators

$$
\dot{x}_{1}(t)=x_{2}(t) ; \ldots ; \dot{x}_{n}(t)=u(t),
$$

is FT-GUAS with the following feedback $u(t)=$ $-\sum_{i=1}^{n} p_{i-1}(t) x_{i}(t)$ where $p(t)=\left(p_{0}(t), \ldots, p_{n-1}(t)\right)$ and $p_{0}, p_{j}(1 \leq j \leq n-1)$ satisfy (13)-(14) with $r_{i}>0, r_{i} \neq r_{j}$ for $i \neq j$ and $c(t)$ given by (2).

\section{PRESCRIBED TIME PREDICTOR CONTROL: PDE BACKSTEPPING APPROACH}

We consider the following LTI system with single input delay:

$$
\dot{z}(t)=A z(t)+B u(t-h), \quad z \in \mathbb{R}^{n}, u \in \mathbb{R},
$$

where $h>0$ is a known constant delay. $(A, B)$ are matrices of appropriate dimensions and satisfy the Kalman rank controllability condition for LTI systems. Thus $(A, B)$ can be assumed to be in the canonical controllability form without loss of generality: this is $A=J_{n}+\mathcal{L}_{n}(a), a=\left(a_{0}, \ldots, a_{n-1}\right)^{\top}, B=$ $(0, \ldots, 0,1)^{\top}$. We assume $u(t)=0, \forall t \in\left[t_{0}-h, t_{0}\right)$.

Our aim is to design a control (predictor-type) for the system (28) which achieves FT-GUAS within a prescribed-time $t_{0}+$ $h+T$ where $T$ is a positive real number that is fixed a priori, $h$ is the known input delay and $t_{0}$ is the initialization time.

To that end, the methodology developed in this paper relies on representing the input delay as a linear transport PDE, and builds on the cascade PDE-ODE setting (i.e. cascade linear hyperbolic PDE with an LTI system) introduced in [28], [27] along with the backstepping approach which makes uses of an invertible Volterra transformation (whose kernels are timevarying). As we will see, the key idea is to transform the original system into suitable target system that is FT-GUAS and that meets the requirements for a convergence in a time $T+h+t_{0}$.

We henceforth represent (28) as cascade PDE-ODE system,

$$
\begin{aligned}
\dot{z}(t) & =A z(t)+B \omega(t, 0), \\
\omega_{t}(t, x) & =\omega_{x}(t, x), \\
\omega(t, h) & =u(t),
\end{aligned}
$$

$t \geq t_{0}, x \in[0, h], w(t, \cdot)$ is the transport PDE state at time $t$ whose solution is given by $\omega(t, x)=u(t+x-h)$ and, in particular, $\omega(t, 0)=u(t-h)$.

We aim at stabilizing (29) (in turn (28) in a prescribed time $t_{0}+T+h$ which is not shorter than the delay. For our analysis and design, we consider the following assumption:

${ }^{2} \exp \left(-r_{\min } \bar{c}_{0} T \sqrt{c(t)}\right)$ is a monotonically decreasing smooth "bump-like $"$ function having the property $\exp \left(-r_{\min } \bar{c}_{0} T \sqrt{c\left(t_{0}+T\right)}\right) \equiv 0$ (see e.g. [37] and [38] for details about the exponential soft landing concept). 
Assumption 1: For a known delay $h$, the prescribed-time $T$ is fixed such that $T \geq h$.

This assumption may constitute a limitation in the control design of our prescribed-time predictor feedback via backstepping. It comes into play in the theoretical proof for the bounded invertibility of the backstepping transformation. For the case $T<h$, one might not guarantee the bounded invertibility of the backstepping transformation and thus the control might not turn out to be bounded either. This issue is further discussed in Section IV

\section{A. Backstepping control design and time-varying kernels}

We build on the backsteeping approach inspired by [27]. Although here, the invertible Volterra integral transformation is chosen to depend on time. It is given as follows:

$$
\begin{gathered}
\zeta(t, x)=\omega(t, x)-\int_{0}^{x} q(t-h, x, y) \omega(t, y) d y \\
-\gamma^{\top}(t-h, x) z(t)
\end{gathered}
$$

such that $\sum_{i=1}^{n} \gamma_{i}(t-h, x) z_{i}(t)=\gamma^{\top}(t-h, x) z(t)$. The kernel function $q$ and the function $\gamma$ are time-varying. Under (30), we want to transform 29) into the following target system:

$$
\begin{aligned}
\dot{z}(t) & =C(t-h) z(t)+B \zeta(t, 0), \\
\zeta_{t}(t, x) & =\zeta_{x}(t, x), \\
\zeta(t, h) & =0
\end{aligned}
$$

with initial condition

$$
\begin{aligned}
\zeta_{0}(x) & =\zeta\left(t_{0}, x\right) \\
& =\omega\left(t_{0}, x\right)-\int_{0}^{x} q\left(t_{0}-h, x, y\right) \omega\left(t_{0}, y\right) d y \\
& -\gamma^{\top}\left(t_{0}-h, x\right) z\left(t_{0}\right) .
\end{aligned}
$$

The time-varying matrix $C(t-h)$ is given by $C(t-h)=$ $J_{n}+\mathcal{L}_{n}(-p(t-h))$, i.e.,

$$
\begin{gathered}
C(t-h)= \\
\left(\begin{array}{cccc}
0 & 1 & \cdots & 0 \\
\vdots & \vdots & \ddots & \vdots \\
0 & 0 & \cdots & 1 \\
-p_{0}(t-h) & -p_{1}(t-h) & \cdots & -p_{n-1}(t-h)
\end{array}\right)
\end{gathered}
$$

and $p_{0}(\cdot), p_{j}(\cdot), j=1 \ldots n-1$ are given by (13), (14), respectively in Proposition 3 By using the results of Lemma 2 along with other issues that we are going to discuss in Section IV] we expect the target system to converge to zero when $t$ tends to $t_{0}+h+T$.

Following the standard methodology to find the kernel equations and taking into account their time-dependence, it can be shown that the kernels of transformation (30) satisfy the following PDE system:

$$
\begin{aligned}
q_{x}(t-h, x, y)+q_{y}(t-h, x, y) & =q_{t}(t-h, x, y), \\
q(t-h, x, 0) & =\gamma^{\top}(t-h, x) B, \\
\gamma_{x}(t-h, x)-I_{n} \gamma_{t}(t-h, x) & =A^{\top} \gamma(t-h, x),
\end{aligned}
$$

where $q$ and $\gamma$ are defined on the domains, respectively

$$
\begin{array}{r}
\mathcal{T}_{q}:\left\{(t, x, y): 0 \leq y \leq x \leq h, t_{0} \leq t<t_{0}+T+h-x\right\} \\
\mathcal{T}_{\gamma}:\left\{(t, x): 0 \leq x \leq h, t_{0} \leq t<t_{0}+T+h-x\right\}
\end{array}
$$

Proposition 4: The system (34) has well-posed $\mathcal{C}^{\infty}$ solutions on $\mathcal{T}_{q}$ and $\mathcal{T}_{\gamma}$, given by

$$
\begin{aligned}
q(t-h, x, y) & =\Gamma^{\top}(t-h+x) \exp (A(x-y)) B, \\
\gamma(t-h, x) & =\exp \left(A^{\top} x\right) \Gamma(t-h+x),
\end{aligned}
$$

where

$\Gamma_{i}(t-h+x)=-\left(a_{i-1}+p_{i-1}(t-h+x)\right), \quad i=1, \ldots, n$,

and functions $p_{i-1}$ are defined by (13), (14).

Proof: By the method of characteristics, the solution of the linear hyperbolic system $\gamma$ in (34) is as follows:

$$
\gamma(t-h, x)=\exp \left(A^{\top} x\right) \Gamma(t-h+x),
$$

where $\Gamma$ is going to be characterized in the sequel. In 30 letting $x=0$ we get $\zeta(t, 0)=\omega(t, 0)-\gamma^{\top}(t-h, 0) z(t)$, which combined with $\dot{z}=A z(t)+B \omega(t, 0)=C(t-h) z(t)+$ $B \zeta(t, 0)$, gives

$A z(t)+B \omega(t, 0)=C(t-h) z(t)+B\left(\omega(t, 0)-\gamma^{\top}(t-h, 0) z(t)\right)$

leading to

$$
B \gamma^{\top}(t-h, 0)=-(A-C(t-h)) .
$$

Now using $A=J_{n}+\mathcal{L}_{n}(a), a=\left(a_{0}, \ldots, a_{n-1}\right)^{\top}, B=$ $(0, \ldots, 0,1)^{\top}, C(t-h)=J_{n}+\mathcal{L}_{n}(-p(t-h))$ given explicitly by (33), we further deduce that

$$
\gamma_{i}(t-h, 0)=-a_{i-1}-p_{i-1}(t-h), \quad i=1, . ., n,
$$

with $p_{i-1}$ computed according to (13), (14). Moreover, it holds, for $i=1, \ldots, n$

$\Gamma_{i}(t-h+x)=\gamma_{i}(t-h+x, 0)=-\left(a_{i-1}+p_{i-1}(t-h+x)\right)$.

Hence, one can realize the solution (38) along with (39). This yields

$$
q(t-h, x, 0)=\Gamma^{\top}(t-h+x) \exp (A x) B .
$$

Consequently,

$$
q(t-h, x, y)=\Gamma^{\top}(t-h+x) \exp (A(x-y)) B,
$$

\section{B. Inverse transformation and time-varying kernels}

The analysis of prescribed-time stability of the closed-loop system requires the study of the inverse transformation which is given by

$$
\begin{gathered}
\omega(t, x)=\zeta(t, x)+\int_{0}^{x} m(t-h, x, y) \zeta(t, y) d y \\
+\bar{\gamma}^{\top}(t-h, x) z(t),
\end{gathered}
$$

whose kernels can be shown to satisfy the following PDE system:

$$
\begin{aligned}
& m_{x}(t-h, x, y)+m_{y}(t-h, x, y)=m_{t}(t-h, x, y), \\
& m(t-h, x, 0)=\bar{\gamma}^{\top}(t-h, x) B, \\
& \bar{\gamma}_{x}(t-h, x)-I_{n} \bar{\gamma}_{t}(t-h, x)=C^{\top}(t-h) \bar{\gamma}(t-h, x),
\end{aligned}
$$


defined on the domains $\mathcal{T}_{m}:\left\{(t, x, y): 0 \leq y \leq x \leq h, t_{0} \leq\right.$ $\left.t<t_{0}+T+h-x\right\}$ and $\mathcal{T}_{\bar{\gamma}}:\left\{(t, x): 0 \leq x \leq h, t_{0} \leq t<\right.$ $\left.t_{0}+T+h-x\right\}$.

Proposition 5: The system (44) has well-posed solutions $\mathcal{C}^{\infty}$ on $\mathcal{T}_{m}$ and $\mathcal{T}_{\bar{\gamma}}$ given by

$m(t-h, x, y)=\Gamma^{\top}(t-h+x) \mathfrak{V}(t-h+x) \mathfrak{V}^{-1}(t-h+y) B,(45)$

$\bar{\gamma}(t-h, x)=\mathfrak{V}^{-\top}(t-h) \mathfrak{V}^{\top}(t-h+x) \Gamma(t-h+x)$,

where

$$
\Gamma_{i}(t-h+x)=-\left(a_{i-1}+p_{i-1}(t-h+x)\right),
$$

with $p_{i-1}$ defined by (13), 14), and $\mathfrak{V}$ is given by (21).

Proof: Following the same reasoning as in the proof of Proposition 4, we will seek first for a solution of $\bar{\gamma}$. To that end, we perform the following change of coordinates:

$$
\bar{\gamma}^{*}(t-h, x)=V^{\top}(t-h) \bar{\gamma}(t-h, x),
$$

where $V$ is the Vandermonde matrix that has been defined in (8) along with (11). Therefore (7) holds at $t-h$ that is $V(t-h) D(t-h)=C(t-h) V(t-h)-\dot{V}(t-h)$ leading to

$$
V^{\top}(t-h) C^{\top}(t-h)-\dot{V}^{\top}(t-h)=D(t-h) V^{\top}(t-h)
$$

Then, $\bar{\gamma}^{*}(t-h, x)$ satisfies the following linear hyperbolic system:

$$
\bar{\gamma}_{x}^{*}(t-h, x)-I_{n} \bar{\gamma}_{t}^{*}(t-h, x)=D(t-h) \bar{\gamma}^{*}(t-h, x),
$$

where $D(t-h)=\operatorname{diag}\left(-r_{1} c(t-h), \ldots,-r_{n} c(t-h)\right)$. Note that we can see (49) as a system of decoupled transport equations whose explicit solutions can be found by the method of characteristics. Indeed, for $i=1, \ldots n$ :

$\bar{\gamma}_{i}^{*}(t-h, x)=\exp \left(-r_{i} \int_{0}^{x} c(t-h+x-s) d s\right) \bar{\Gamma}_{i}^{*}(t-h+x)$.

Since $\int_{0}^{x-y} D(t-h+x-s) d s=\int_{t-h+y}^{t-h+x} D(s) d s$ and

$$
\begin{aligned}
\exp \left(\int_{t-h+y}^{t-h+x} D(s) d s\right) & =\mathfrak{D}(t-h+x) \mathfrak{D}^{-1}(t-h+y) \\
& =\mathfrak{D}^{-1}(t-h+y) \mathfrak{D}(t-h+x),
\end{aligned}
$$

(see (16) to recall the notation of $\mathfrak{D}$ ) then, setting $y=0$, we have in compact form

$$
\bar{\gamma}^{*}(t-h, x)=\mathfrak{D}^{-1}(t-h) \mathfrak{D}(t-h+x) \bar{\Gamma}^{*}(t-h+x),
$$

where $\bar{\Gamma}^{*}$ is yet to be characterized.

On the other hand, we use the inverse transformation at $x=0$ such that $\omega(t, 0)=\zeta(t, 0)+\bar{\gamma}^{\top}(t-h, 0) z(t)$ combined with $\dot{z}(t)=A z(t)+B \omega(t, 0)=C(t-h) z(t)+B \zeta(t, 0)$ which gives

$$
B \bar{\gamma}^{\top}(t-h, 0)=-(A-C(t-h)),
$$

and using again $A=J_{n}+\mathcal{L}_{n}(a), a=\left(a_{0}, \ldots, a_{n-1}\right)^{\top}, B=$ $(0, \ldots, 0,1)^{\top}, C(t-h)$ given by (33), we deduce that:

$$
\bar{\gamma}_{i}(t-h, 0)=-a_{i-1}-p_{i-1}(t-h), \quad i=1, . ., n .
$$

Under (47) and using the structure of the Vandermonde matrix (8), we have

$$
\begin{aligned}
& \bar{\gamma}_{i}^{*}(t-h, 0)=\left(-a_{0}-p_{0}(t-h)\right) \\
& \quad+\sum_{k=2}^{n}\left(-a_{k-1}-p_{k-1}(t-h)\right)\left(\delta^{k-2}\left(-r_{i} c\right)\right)(t-h) .
\end{aligned}
$$

Therefore from (56), it holds

$$
\bar{\Gamma}_{i}^{*}(t-h+x)=\bar{\gamma}_{i}^{*}(t-h+x, 0) .
$$

Note that $\bar{\Gamma}^{*}=V^{\top} \Gamma$ with $\Gamma$ obtained in (39). Hence, (53) in conjunction with (57) give the solution to the linear hyperbolic system (49). Therefore, by virtue of (47) together with $\mathfrak{V}(s)=$ $V(s) \mathfrak{D}(s)$, we obtain

$$
\bar{\gamma}(t-h, x)=\mathfrak{V}^{-\top}(t-h) \mathfrak{V}^{\top}(t-h+x) \Gamma(t-h+x) .
$$

It yields

$m(t-h, x, 0)=\Gamma^{\top}(t-h+x) \mathfrak{V}(t-h+x) \mathfrak{V}^{-1}(t-h) B$,

from which, we can obtain

$m(t-h, x, y)=\Gamma^{\top}(t-h+x) \mathfrak{V}(t-h+x) \mathfrak{V}^{-1}(t-h+y) B$.

This concludes the proof.

\section{Prescribed-time predictor control}

From (30), and using (37)-38 at $x=h$, the boundary control is then

$$
\begin{aligned}
u(t)= & \Gamma^{\top}(t) \exp (A h) z(t) \\
& +\int_{0}^{h} \Gamma^{\top}(t) \exp (A(h-y)) B u(t-h+y) d y,
\end{aligned}
$$

where $u(t-h+y)=w(t, y)$. Using the inverse transformation, from (43), and using (45)- 46) at $x=h$, the boundary control can equivalently be written as follows:

$$
\begin{aligned}
u(t)= & \Gamma^{\top}(t) \mathfrak{V}(t) \mathfrak{V}^{-1}(t-h) z(t) \\
& +\int_{0}^{h} \Gamma^{\top}(t) \mathfrak{V}(t) \mathfrak{V}^{-1}(t-h+y) B \zeta(t, y) d y .
\end{aligned}
$$

This equivalent form is instrumental for our analysis. Indeed, under this form we are able to establish the boundedness of the controller and its convergence to zero. As we will see, we take advantage of the transport PDE $\zeta$ which vanishes after $h$ units of time, thus greatly simplifying the analysis.

\section{STABility ANALYSis}

We perform the stability analysis on the target system and then we establish the bounded invertibility of the transformations under a suitable norm equivalence.

Lemma 3: Let $C(t-h)=J_{n}+\mathcal{L}_{n}(-p(t-h))$ be given as in (33) with $p_{i-1}(i=1, \ldots, n)$ defined by (13), (14), and $c$ defined by (2). Then, for any $z_{0} \in \mathbb{R}^{n}$, there exist $\eta_{z}>0$ and positive polynomials $P$ and $Q$ in $\sqrt{c(t-h)}$ such that the solution $z$-dynamics of (31) satisfies, for all $t \in\left[t_{0}, t_{0}+h+T\right.$ )

$$
\begin{aligned}
\|z(t)\|^{2} & \leq \mathfrak{V}_{-r_{\min }}(t-h) \\
& \times\left(\bar{a}(t-h)\left\|z\left(t_{0}\right)\right\|^{2}+\bar{b}\left(t-h, t_{0}\right)\left\|\zeta_{0}\right\|_{\left[t_{0}, t\right]}^{2}\right),
\end{aligned}
$$


where

$$
\begin{aligned}
\bar{a}(t-h) & =2 \eta_{z} P(\sqrt{c(t-h)}), \\
\bar{b}\left(t-h, t_{0}\right) & =Q(\sqrt{c(t-h)}) \int_{t_{0}}^{t} \frac{\mathfrak{e}_{2 r_{\max }}(\tau-h)}{c(\tau-h)^{2 n-2}} d \tau,
\end{aligned}
$$

where $\left\|\zeta_{0}\right\|_{\left[t_{0}, t\right]}=\sup _{t_{0} \leq \tau \leq t}\left|\zeta_{0}(\tau)\right|$. In particular, it holds

$$
\|z(t)\| \rightarrow 0, \quad t \rightarrow t_{0}+h+T .
$$
(5):

Proof: Consider the following change of variables as in

$$
\bar{z}(t)=V^{-1}(t-h) z(t),
$$

with $V(\cdot)$ given by (8), (11) (in light of Proposition 2). Therefore, the target system (31) is transformed into the following system:

$$
\begin{aligned}
\dot{\bar{z}}(t) & =D(t-h) \bar{z}(t)+V^{-1}(t-h) B \zeta(t, 0), \\
\zeta_{t}(t, x) & =\zeta_{x}(t, x), \\
\zeta(t, h) & =0,
\end{aligned}
$$

where $D(t-h)=V^{-1}(t-h) C(t-h) V(t-h)-$ $V^{-1}(t-h) \dot{V}(t-h)$ which gives specifically $D(t-h)=$ $\operatorname{diag}\left(-r_{1} c(t-h), \ldots,-r_{n} c(t-h)\right)$ (see Section [I-B). The solution of the $\bar{z}$-dynamics of (65) is given as follows:

$$
\begin{aligned}
\bar{z}(t)= & \bar{\Phi}_{D}\left(t-h, t_{0}-h\right) \bar{z}\left(t_{0}\right) \\
& +\int_{t_{0}}^{t} \bar{\Phi}_{D}(t-h, \tau-h) V^{-1}(\tau-h) B \zeta(\tau, 0) d \tau
\end{aligned}
$$

where $\bar{\Phi}_{D}\left(t-h, t_{0}-h\right)=\exp \left(\int_{t_{0}}^{t} D(s-h) d s\right)=$ $\exp \left(\int_{t_{0}-h}^{t-h} D(s) d s\right)$ is the state transition matrix of the underlying linear (diagonal-form) time-varying system $(\bar{z}$ dynamics). Using $\bar{\Phi}_{D}\left(t-h, t_{0}-h\right)=\mathfrak{D}(t-h) \mathfrak{D}^{-1}\left(t_{0}-h\right)$ (as in (18) with $\mathfrak{D}$ given by (16)), we get

$$
\begin{aligned}
& \bar{z}(t)=\mathfrak{D}(t-h) \mathfrak{D}^{-1}\left(t_{0}-h\right) \bar{z}\left(t_{0}\right) \\
& +\int_{t_{0}}^{t} \mathfrak{D}(t-h) \mathfrak{D}^{-1}(\tau-h) V^{-1}(\tau-h) B \zeta(\tau, 0) d \tau
\end{aligned}
$$

Then, from (64), along with (20) and (21), the solution of the the $z$-dynamics of (31) is given, for all $t \in\left[t_{0}, t_{0}+h+T\right)$, as follows:

$$
\begin{aligned}
z(t)= & \Phi_{C}\left(t-h, t_{0}-h\right) z\left(t_{0}\right) \\
& +\int_{t_{0}}^{t} \Phi_{C}(t-h, \tau-h) B \zeta(\tau, 0) d \tau,
\end{aligned}
$$

where $\Phi_{C}\left(t-h, t_{0}-h\right)=\mathfrak{V}(t-h) \mathfrak{V}^{-1}\left(t_{0}-h\right)$. We denote

$$
\Phi_{C}^{B}(t-h, \tau-h)=\Phi_{C}(t-h, \tau-h) B,
$$

which corresponds to the $n$-th column vector of the matrix $\Phi_{C}(t-h, \tau-h)$. Using the structure of $V$ and $V^{-1}$ (see (11) along with (120)- (124) in Appendix C for more information about the characterization), we assert that each component of the vector $\Phi_{C}^{B}(t-h, \tau-h)$ has the following form:

$$
\begin{aligned}
\Phi_{C 1}^{B}(t-h, & \tau-h)=\frac{1}{(c(\tau-h))^{n-1}} \sum_{l=1}^{n}(-1)^{l} \mathcal{R}_{l}\left(r_{l}\right) \\
& \times \mathfrak{e}_{-r_{l}}(t-h) \mathfrak{e}_{r_{l}}(\tau-h),
\end{aligned}
$$

and for all $k=2, \ldots, n$

$$
\begin{aligned}
& \Phi_{C k}^{B}(t-h, \tau-h)=\frac{1}{(c(\tau-h))^{n-1}} \sum_{l=1}^{n}(-1)^{k+l} \mathcal{R}_{l}\left(r_{l}\right) \\
& \left.\times \delta^{k-2}\left(-r_{l} c\right)\right)(t-h) \mathfrak{e}_{-r_{l}}(t-h) \mathfrak{e}_{r_{l}}(\tau-h),
\end{aligned}
$$

where $\mathcal{R}_{l}\left(r_{l}\right)$ is some rational function of $r_{1}, \ldots, r_{n}$ whose specific characterization is not important for the current analysis. Recall $\left(\delta^{k-2}\left(-r_{l} c\right)\right)(t-h)=(k-$ $2) ! \mathfrak{s}_{-r_{l}}^{k-2}(t) L_{k-2}^{(1)}\left(\mathfrak{s}_{r_{l}}^{-1}(t-h)\right)$ in light of formulas (11), (12) and also that $\left|L_{k-2}^{(1)}\left(\mathfrak{s}_{r_{l}}^{-1}(t-h)\right)\right| \leq\left(\begin{array}{c}k-1 \\ k-2\end{array}\right) \mathfrak{e}_{\frac{r_{l}}{2}}(t-h)$. Therefore, from (68) and inspired by (17) in Lemma 2, the following estimate holds, for all $t \in\left[t_{0}, t_{0}+h+T\right)$ :

$$
\begin{aligned}
\|z(t)\|^{2} \leq & \mathfrak{r}_{-r_{\min }}(t-h) \bar{a}(t-h)\left\|z\left(t_{0}\right)\right\|^{2} \\
& +\tilde{b}\left(t-h, t_{0}\right)\|\zeta(\tau, 0)\|_{\left[t_{0}, t\right]}^{2},
\end{aligned}
$$

with

$$
\tilde{b}\left(t-h, t_{0}\right)=2 n \max _{1 \leq k \leq n}\left\{\int_{t_{0}}^{t}\left|\Phi_{C k}^{B}(t-h, \tau-h)\right| d \tau\right\}^{2},
$$

and $\bar{a}(t-h)$ is given in (63). Let us find a more tractable estimate of $\tilde{b}\left(t-h, t_{0}\right)$.

By virtue of the characterizations (70) and (71), it holds

$\int_{t_{0}}^{t}\left|\Phi_{C 1}^{B}(t-h, \tau-h) d \tau\right| \leq \mathfrak{e}_{\frac{-r_{\min }}{2}}(t-h) \int_{t_{0}}^{t} \frac{\mathfrak{e}_{r_{\max }}(\tau-h)}{c(\tau-h)^{n-1}} d \tau$,

and for $k=2, \ldots, n$,

$$
\begin{aligned}
& \int_{t_{0}}^{t}\left|\Phi_{C k}^{B}(t-h, \tau-h) d \tau\right| \leq \bar{P}_{k}(\sqrt{c(t-h)}) \\
& \quad \times \mathfrak{e}_{\frac{-r_{\min }}{2}}(t-h) \int_{t_{0}}^{t} \frac{\mathfrak{e}_{r_{\max }}(\tau-h)}{c(\tau-h)^{n-1}} d \tau
\end{aligned}
$$

where $\bar{P}_{k}(\sqrt{c(t-h)})=n(k-1) ! \overline{\mathcal{R}}_{-r_{\text {max }}}^{k-2}(t-h)$ and $\overline{\mathcal{R}}=$ $\max _{1 \leq l \leq n}\left\{\left|\mathcal{R}_{l}\left(r_{l}\right)\right|\right\}$ (whose specific characterization is not needed in the analysis). This leads to $\tilde{b}\left(t-h, t_{0}\right) \leq \bar{b}(t-$ $\left.h, t_{0}\right)=\mathfrak{e}_{-r_{\text {min }}}(t-h) Q(\sqrt{c(t-h)}) \int_{t_{0}}^{t} \frac{\mathfrak{e}_{2 r_{\max }(\tau-h)}}{c(\tau-h)^{2 n-2}} d \tau$ with

$$
Q(\sqrt{c(t-h)})=4 n^{2} \max _{1 \leq k \leq n}\left\{1, \bar{P}_{k}^{2}(\sqrt{c(t-h)})\right\} .
$$

Thus (62) holds for $t \in\left[t_{0}, t_{0}+h+T\right)$. We exploit the cascade nature of the chosen target system (31) along with the fact that the transport PDE $\zeta$ is fixed-time stable. Indeed, by virtue of the method of characteristics, $\zeta(t, x)=\zeta_{0}(x+t)$ for $t \leq$ $t_{0}+h-x$ and $\zeta(t, x)=0$ for $t \geq t_{0}+h-x$. Thus, we have an analytical expression for $\zeta(t, 0)$; that is $\zeta(t, 0)=\zeta_{0}(t)$ for $t \leq t_{0}+h$. In addition, after $t=t_{0}+h$, one has $\zeta(t, 0)=0$. Let us then refine the analysis by subdividing $\left[t_{0}, t_{0}+h+T\right)$ into two sub-intervals. First, we consider $t \in\left[t_{0}, t_{0}+h\right]$. From (72) and analyzing (74) and (75), one can infer that the last term of (72) remains bounded. This is still valid irrespective of $T<h$ or $T \geq h$ because finite-time escape of the last term in (72) may occur only after $t=t_{0}+h$. Notice, in addition, that it holds that $\left\|\zeta_{0}(\tau)\right\|_{\left[t_{0}, t\right]} \leq\left\|\zeta_{0}(\tau)\right\|_{\left[t_{0}, t_{0}+h\right]}<\infty$. Hence, we can conclude that, for all $t \in\left[t_{0}, t_{0}+h\right],\|z(t)\|^{2}<\infty$, thus $\left\|z\left(t_{0}+h\right)\right\|<\infty$. Then, we consider the case when 
$t \in\left[t_{0}+h, t_{0}+h+T\right)$. Using the fact that $\zeta \equiv 0$ for $t \geq t_{0}+h$, we get

$$
\|z(t)\|^{2} \leq \mathfrak{e}_{-r_{\min }}(t-h) \bar{a}(t-h)\left\|z\left(t_{0}+h\right)\right\|^{2} .
$$

Finally, by following the same arguments as in the proof of Lemma2, we can conclude that $\|z(t)\|^{2} \rightarrow 0$ as $t \rightarrow t_{0}+h+T$.

Proposition 6: For the backstepping transformations (30) and (43) along with (37)-(38) and (45)-(46), the following estimates hold:

$$
\begin{gathered}
\|\zeta(t, \cdot)\|_{L^{2}}^{2} \leq M_{q}(t-h)\|\omega(t, \cdot)\|_{L^{2}}^{2}+M_{\gamma}(t-h)\|z(t)\|^{2}, \\
\|\omega(t, \cdot)\|_{L^{2}}^{2} \leq N_{m}(t-h)\|\zeta(t, \cdot)\|_{L^{2}}^{2}+N_{\bar{\gamma}}(t-h)\|z(t)\|^{2} \\
M_{q}(t-h)=4\left(1+\int_{0}^{h}\left(\int_{0}^{x}|q(t-h, x, y)|^{2} d y\right) d x\right) \\
M_{\gamma}(t-h)=2 \int_{0}^{h} \gamma^{\top}(t-h, x) \gamma(t-h, x) d x \\
N_{m}(t-h)=4\left(1+\int_{0}^{h}\left(\int_{0}^{x}|m(t-h, x, y)|^{2} d y\right) d x\right) \\
N_{\bar{\gamma}}(t-h)=2 \int_{0}^{h} \bar{\gamma}^{\top}(t-h, x) \bar{\gamma}(t-h, x) d x
\end{gathered}
$$

Proof: It follows by virtue of the triangle, Young's and Cauchy-Schwarz inequalities applied to (30) and (43).

Lemma 4: Let $N_{\bar{\gamma}}(t-h)$ be given by 83 with $\bar{\gamma}$ given by (46) and let $P(\sqrt{c(t-h)})$ as in Lemma 3 . Then, the following holds true:

$$
\lim _{t \rightarrow t_{0}+h+T} N_{\bar{\gamma}}(t-h) P(\sqrt{c(t-h)}) \mathfrak{e}_{-r_{\min }}(t-h)=0 .
$$

\section{Proof: See Appendix D}

Theorem 1: Let $h>0$ be a known delay and $T>0$ a fixed time such that $T \geq h$ (under Assumption 1). Let $c(t-h)$ given by (2). Let $\eta_{z}$ and $P(\sqrt{c(t-h)})$ be as in Lemma 3 and $N_{\bar{\gamma}}(t-h)$ be given by (83). Then, the solution of the closedloop system 29) with the prescribed-time predictor feedback control (60) (or 61) is such that, for any $z_{0} \in \mathbb{R}^{n}$, for all $t \in\left[t_{0}, t_{0}+h\right],\|z(t)\|^{2}+\|\omega(t, \cdot)\|_{L^{2}}^{2}$ remains bounded and for all $t \in\left[t_{0}+h, t_{0}+h+T\right)$,

$$
\begin{aligned}
\|z(t)\|^{2}+ & \|\omega(t, \cdot)\|_{L^{2}}^{2} \leq \eta_{z}\left(1+N_{\bar{\gamma}}(t-h)\right) \\
& \times P(\sqrt{c(t-h)}) \mathfrak{e}_{-r_{\min }}(t-h)\left\|z_{0}\right\|^{2} .
\end{aligned}
$$

In particular,

$$
\|z(t)\|^{2}+\|\omega(t, \cdot)\|_{L^{2}}^{2} \rightarrow 0, \quad \text { as } \quad t \rightarrow t_{0}+h+T,
$$

and $|u(t)| \rightarrow 0$ as $t \rightarrow t_{0}+T$.

Proof: Using the norm equivalences in Proposition 6 it holds, for $t \in\left[t_{0}, t_{0}+h+T\right)$ that

$$
\begin{gathered}
\|z(t)\|^{2}+\|\omega(t, \cdot)\|_{L^{2}}^{2} \leq N_{m}(t-h)\|\zeta(t, \cdot)\|_{L^{2}}^{2} \\
+\left(1+N_{\bar{\gamma}}(t-h)\right)\|z(t)\|^{2} .
\end{gathered}
$$

Let us focus first on $N_{m}(t-h)$ which is given by (82). Notice that $m(t-h, x, y)$ given by (45) can be written as follows:

$$
\begin{aligned}
m(t-h, x, y)= & -\sum_{i=1}^{n}\left(a_{i-1}+p_{i-1}(t-h+x)\right) \\
& \times \Phi_{C i}^{B}(t-h+x, t-h+y),
\end{aligned}
$$

where we make use again of expressions (69)-(71) which allow to get the following estimate:

$$
\begin{aligned}
& |m(t-h, x, y)|^{2} \leq \bar{Q}(\sqrt{c(t-h+x)}) \\
& \times \mathfrak{\ell}_{-r_{\min }}(t-h+x) \frac{\mathfrak{\mathfrak { e }}_{2 r_{\max }}(t-h+y)}{c(t-h+y)^{2 n-2}},
\end{aligned}
$$

with $\bar{Q}(\sqrt{c(t-h+x)})$ being a positive polynomial function (whose specific characterization is not needed in this analysis). Then, from (82) and under Assumption 1 (i.e. $T \geq h$ ), we have that the integral term,

$$
\begin{array}{r}
\int_{0}^{h}\left(\bar{Q}(\sqrt{c(t-h+x)}) \mathfrak{e}_{-r_{\min }}(t-h+x)\right. \\
\left.\times \int_{0}^{x} \frac{\mathfrak{e}_{2 r_{\max }(t-h+y)}}{c(t-h+y)^{2 n-2}} d y\right) d x<\infty,
\end{array}
$$

for all $t \in\left[t_{0}, t_{0}+h\right]$. From which, we deduce that for all $t \in\left[t_{0}, t_{0}+h\right],\|z(t)\|^{2}+\|\omega(t, \cdot)\|_{L^{2}}^{2}$ remains bounded. Finitetime escape for the above integral term may occur only at $t=$ $t_{0}+h+T$. Nevertheless, we use the fact that $\|\zeta(t, \cdot)\|_{L^{2}} \equiv 0$ for all $t \geq t_{0}+h$. Hence, the term $N_{m}(t-h)\|\zeta(t, \cdot)\|_{L^{2}}^{2}$ remains bounded for all $t \in\left[t_{0}, t_{0}+h+T\right)$.

Now, by virtue of Lemma 3, it holds for all $t \in\left[t_{0}, t_{0}+\right.$ $h+T)$

$$
\begin{aligned}
\|z(t)\|^{2} & +\|\omega(t, \cdot)\|_{L^{2}}^{2} \leq N_{m}(t-h)\|\zeta(t, \cdot)\|_{L^{2}}^{2} \\
+ & \eta_{z}\left(1+N_{\bar{\gamma}}(t-h)\right) P(\sqrt{c(t-h)}) \\
& \times \mathfrak{R}_{-r_{\min }}(t-h)\left\|z\left(t_{0}\right)\right\|^{2} \\
+ & \left(1+N_{\bar{\gamma}}(t-h)\right) Q(\sqrt{c(t-h)}) \mathfrak{e}_{-r_{\min }}(t-h) \\
& \times \int_{t_{0}}^{t} \frac{\mathfrak{\mathfrak { e }}_{2 r_{\max }}(\tau-h)}{c(\tau-h)^{2 n-2}} d \tau \sup _{t_{0} \leq \tau \leq t}|\zeta(\tau, 0)|^{2} .
\end{aligned}
$$

so that, in conjunction with Lemma 4, we finally obtain that $\|z(t)\|^{2}+\|\omega(t, \cdot)\|_{L^{2}}^{2} \rightarrow 0$, as $t \rightarrow t_{0}+h+T$.

It remains to prove that the control remains bounded. Indeed, from 61, the following estimate holds, for all $t \in$ $\left[t_{0}, t_{0}+h+T\right)$ :

$$
\begin{aligned}
|u(t)|^{2} \leq\left\|\Gamma^{\top}(t)\right\|^{2}\left\|\Phi_{C}(t, t-h)\right\|^{2}\|z(t)\|^{2} \\
+\|m(t-h, h, \cdot)\|_{L^{2}}^{2}\|\zeta(t, \cdot)\|_{L^{2}}^{2} .
\end{aligned}
$$

By Lemma 3, we have that $\left\|\Gamma^{\top}(t)\right\|^{2}\left\|\Phi_{C}(t, t-h)\right\|^{2}\|z(t)\|^{2}<$ $\infty$ for all $t \in\left[t_{0}, t_{0}+h+T\right)$. In fact, by following similar arguments as in the proof of Lemma 3 , it can be further shown that $\left\|\Gamma^{\top}(t)\right\|^{2}\left\|\Phi_{C}(t, t-h)\right\|^{2}\|z(t)\|^{2} \rightarrow 0$ as $t \rightarrow t_{0}+T$.

On the other hand, under Assumption 1 (i.e. $T \geq h$ ), we can guarantee that the term $\|m(t-h, h, \cdot)\|_{L^{2}}^{2}<\infty$ for all $t \in\left[t_{0}, t_{0}+h\right)$. Indeed, notice that due to (87)- (88), the following estimate holds:

$$
\begin{aligned}
\| m(t-h, h, \cdot) & \|_{L^{2}}^{2} \leq \bar{Q}(\sqrt{c(t)}) \\
& \times \mathfrak{e}_{-r_{\min }}(t) \int_{0}^{h} \frac{\mathfrak{e}_{2 r_{\max }}(t-h+y)}{c(t-h+y)^{2 n-2}} d y .
\end{aligned}
$$

Finite-time escape for the term in the right-hand side of 91 may occur when $t \rightarrow t_{0}+T$. Nevertheless, we use again the fact that $\|\zeta(t, \cdot)\|_{L^{2}} \equiv 0$ for all $t \geq t_{0}+h$ and the assumption that $T \geq h$. We conclude then that $|u(t)|^{2} \rightarrow 0$ as $t \rightarrow t_{0}+T$. This concludes the proof. 


\section{Discussion about the Assumption 1$]$}

We have stated that the fixed time $T$ should be larger than the known delay $h$. This constraint is related to some issues arising when establishing the bounded invertibility of the backstepping transformation. Indeed, note that in (91) and for all $t \in\left[t_{0}, t_{0}+h\right]$, if we considered $T<h$, then, we would have finite time escape at $t=t_{0}+T$ and the term $\|m(t-h, h, \cdot)\|_{L^{2}}^{2}\|\zeta(t, \cdot)\|_{L^{2}}^{2}$ in (90) may not be bounded nor decreasing to zero. In fact, even tough $\|\zeta(t, \cdot)\|_{L^{2}} \rightarrow 0$ as $t \rightarrow$ $t_{0}+h$, we cannot assess the decreasing-in-time of $\|\zeta(t, \cdot)\|_{L}^{2}$ (due to the lack of a Lyapunov functional from which one can characterize fixed-time convergence property of the transport $\zeta$ - PDE equation) in order to compare with the growth-intime of $\|m(t-h, h, \cdot)\|_{L^{2}}^{2}$. Similar arguments apply for the evolution of $N_{m}(t-h)$ (see (86) and (89) which is essential in the bounded invertibility of the transformation. Consequently, we have opted for considering Assumption 1 in our approach. Relaxing this assumption is still an open and challenging question that might require better (less conservative) estimates of all underlying norms of the transition matrices we have used in the stability analysis. Perhaps, while relaxing this assumption, one may encounter conditions relating $r_{i}, \bar{c}_{0}$ and $T$ whenever a delay $h$ is large and one chooses $T<<1$ very small.

\section{Simulations}

Motivated by guidance, navigation and control applications, we consider the scenario in which a quadrotor Unmanned Aerial Vehicle $(U A V)$ aims at tracking and landing on a moving landing platform. Moreover, it is required that the UAV achieves such objectives within a finite amount of time (that can be prescribed in the design). Under the scenario that the UAV is near the moving landing platform, as well as some other reasonable assumptions ${ }^{3}$, we consider a planar kinematic model that can be linearized and hence approximated by a double integrator, i.e., $\dot{z}_{1}(t)=z_{2}(t), \dot{z}_{2}=u(t-h)$ where $z_{1}$ and $z_{2}$ represent, respectively, the relative position (distance of moving target relative to the UAV perpendicular to the Line of Sight $(L O S)$ ) and the relative velocity. The control $u$ is the relative acceleration (perpendicular to the LOS) which is subject to a small delay. The initial conditions of the relative position and velocity, are $z(0)=(-2[\mathrm{~m}],-2[\mathrm{~m} / \mathrm{s}])^{\top}$ where the initialization time has been set $t_{0}=0$. The command acceleration is subject to a delay $h=0.9[\mathrm{~s}]$. We fix $T=3[\mathrm{~s}]$ so that the UAV is required to land on the moving platform within a prescribed time $T+h=3.9[\mathrm{~s}]$.

For the control design, we use the blow-up function $c(t)$ given in (2) with $\bar{c}_{0}=0.8$ (dimensionless). The timevarying gains involved in the controller (60) and its equivalent form (61), are computed, respectively, from (37)- 38 and (45)-(46), at $x=h$ where $\Gamma(t)=\left(-p_{0}(t),-p_{1}(t)\right)^{\top}$ is computed from (39) and $p_{0}(t)=r_{1} r_{2} c^{2}(t)\left[\mathrm{s}^{-2}\right]$ with

\footnotetext{
${ }^{3}$ Assume that the moving target is nonmanuevering and has a constant velocity. In addition, the UAV has perfect autopilot dynamics and has the capability to track and provide real time position and velocity of the moving target and the UAV itself. We assume that the angle of Line of Sight (LOS) remains small relative to its initial value.
}

$p_{1}(t)=\left(\left(r_{1}+r_{2}\right) c(t)-\frac{2}{\bar{c}_{0} T} \sqrt{c(t)}\right)\left[\mathrm{s}^{-1}\right]$ (obtained according to (13)-(14) and we choose $r_{1}=1\left[\mathrm{~s}^{-1}\right]$ and $r_{2}=2\left[\mathrm{~s}^{-1}\right]$. Figure 1 shows the evolution of the aforementioned gains involved in the controller (60) and (61). Notice that $\bar{\gamma}(t-h, h)$ converges to zero within a fixed time. This feature has also been instrumental for the analysis of the boundedness and convergence in a fixed-time of the controller while using the equivalent form i.e., 61). Numerical simulations are done by discretizing the cascade PDE-ODE systems 29. and (31). For that purpose we have used a two-step variant of the Lax-Friedrichs numerical method presented in [36] and the respective solver on Matlab. The parameters of the numerical scheme are selected so that the Courant-FriedrichLevy condition for the numerical stability holds.

Figure 2 shows the numerical solution of the double integrator with constant input delay, i.e., the closed-loop system (29). More precisely, on the left we can observe the evolution of the states $z_{1}$ and $z_{2}$ of the double integrator when we stabilize with the prescribed-time predictor control 60) (depicted in blue line) as well as the the evolution of the delayed control input (depicted in black line) ${ }^{4}$. On the right we can observe the numerical solution of the transport PDE $\omega(t, x)$ in 29) representing the infinite dimensional actuator dynamics. Figure 3 shows Evolution of $\|z(t)\|^{2}+\|\omega(t, \cdot)\|_{L^{2}}^{2}$ of the closedloop system (29) with the prescribed-time predictor control (60). The plot is in logarithmic scale to better illustrate that the closed-loop system converges in a prescribed time given by $T+h=3.9[\mathrm{~s}]$. It implies that the relative position $z_{1}$ and relative velocity $z_{2}$ convergence to zero, thus implying that the UAV tracks and lands on the moving target with precision and within a prescribed-time $T+h=3.9$ [s]. Finally, we run simulations for two different initial positions of the UAV (which translates in two different initial conditions for the double integrator). Figure 4 shows the trajectories of the UAV (in the Cartesian inertial frame $X Y$ ) when tracking and then landing on the moving target (which is moving to its initial position to the right with constant speed $1.5[\mathrm{~m} / \mathrm{s}]$ ). We compare the cases when the actuator is delay-free (i,e., $h=0[\mathrm{~s}])$ and when is subject to a small delay $h=0.9[\mathrm{~s}]$. For the delay-free case, the landing occurs at prescribed-time $T=3[\mathrm{~s}]$ whereas for the delayed input case, the landing is at $T+h=3.9[\mathrm{~s}]$. In both cases, the prescribed-time convergence is achieved irrespective of the initial conditions of the problem.

\section{Discussion about numerical implementation issues}

Some numerical issues may arise in the implementation that can be caused by the unbounded gains as $t$ approaches to $T+h$. Some ways to overcome the numerical problems in the framework of prescribed-time stabilization have been discussed in [37], [26] and [19]. We follow in particular [19.

\footnotetext{
${ }^{4}$ In Figure 2 we have included the numerical solution of the double integrator when the input is subject to a slowly time-varying delay $h(t)=$ $0.9+0.1 \sin (2 \pi t)$ [s]. The aim of including this, is to observe whether the proposed approach and the resulting predictor feedback might be robust to time-varying delays or not. Simulations show that it may be possible to guarantee robustness and suggest that new theoretical developments, in the framework of PDE-ODE for time-varying delays, need to be developed to fully compensate arbitrary time-varying input delays in prescribed-time. The theoretical development is object of a future work.
} 

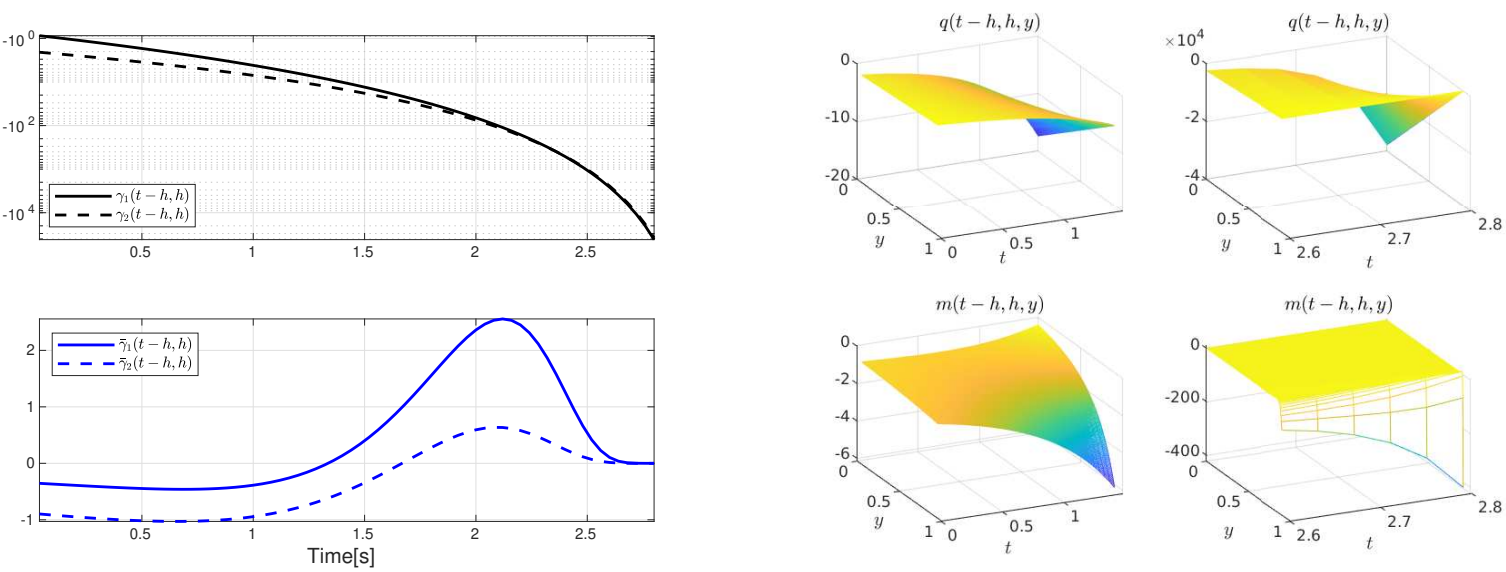

Fig. 1. On the left: evolution of the time-varying gains $\gamma$ (plotted in logaritmic scale) in 38 and $\bar{\gamma}$ in 46 at $x=h$. On the right: evolution of the time-varying kernel gains $q$ in 37] and $m$ in 45 at $x=h$, on two different time intervals.


Fig. 2. On the left: evolution of the states of the double integrator with input delay $(h=0.9[\mathrm{~s}])$ (blue lines), and slowly time-varying delay $(h(t)=$ $0.9+0.1 \sin (2 \pi t)[\mathrm{s}])$ (red lines) when stabilizing with the prescribed-time predictor control 60 (black line). On the right: numerical solution of the transport $\operatorname{PDE} \omega(t, x)$ in 29 .

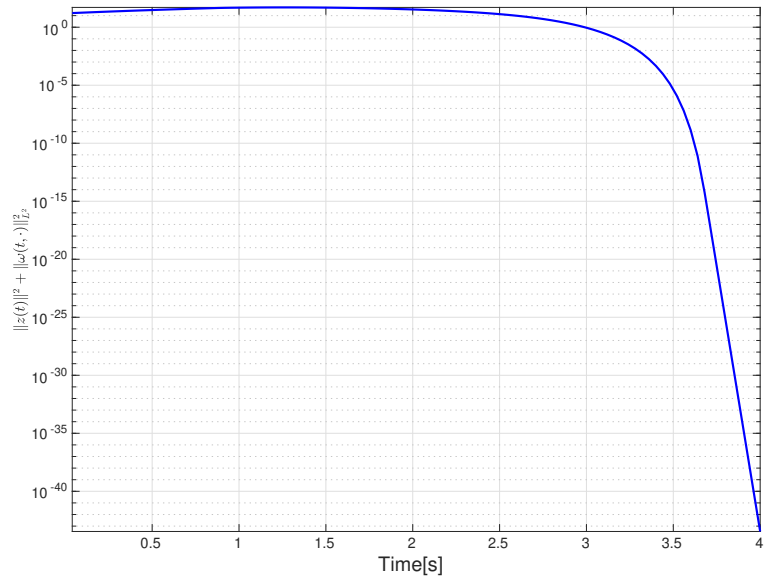

Fig. 3. Evolution of $\|z(t)\|^{2}+\|\omega(t, \cdot)\|_{L^{2}}^{2}$ (plotted in logarithmic scale) of the closed-loop system with the prescribed-time predictor control 60 where $h=0.9[\mathrm{~s}]$ and $T=3 \mathrm{~s}$.
Section II A] in order to make saturation on the control gains. The idea is to monitor the growth of the blow-up function and saturate the control gains to some maximum value when e.g. $t=\epsilon(T+h)$ (with $\epsilon \rightarrow 1$ chosen by the user: $\epsilon=0.95$ in the conducted simulations). Subsequently, we may switch to a classical predictor feedback (whose gains are the latest highest gains of the prescribed-time predictor control before saturation). By doing this, of course, the convergence will not be exactly to origin but to a neighborhood that can be made smaller if desired. In addition, if needed, the prescribed-time predictor control can be reset to begin from a new initial time $t_{n e w}=\epsilon(T+h)$ and with initial conditions that are nearly zero.

It is worth mentioning that some emerging approaches may deal with the issues of implementation and robustness to external disturbance or unmatched uncertainties in a very elegant way. Indeed, one can reformulate the problem, through a suitable time-scaling transformation (see e.g. [40]), to then build on control designs such us homogeneity and sliding 




Fig. 4. Trajectories of the UAV when tracking and then landing on the moving target (which is moving from its initial position to the right with constant speed $1.5[\mathrm{~m} / \mathrm{s}])$. Two different initial positions in the frame $\mathrm{XY}$ are considered; namely $(0[\mathrm{~m}], 2[\mathrm{~m}])$ and $(10[\mathrm{~m}], 2[\mathrm{~m}])$, and with initial speed $2[\mathrm{~m} / \mathrm{s}]$ along the $\mathrm{Y}$ axis. Besides, two cases for the actuation are considered: the control action is i) delay-free and ii) subject to a delay $h=0.9[\mathrm{~s}]$. For the delay-free case, the landing occurs at prescribed-time $T=3[\mathrm{~s}]$ whereas for the input delay case, the landing is at $T+h=3.9[\mathrm{~s}]$.

mode feedbacks. Other approaches include switched linear feedback with state-dependent switching laws when approaching the terminal time or the use of state-dependent cut-off functions inspired by what is done in the context of $1 \mathrm{D}$ reaction-diffusion PDEs [12].

\section{CONCLUSION}

In this paper we have addressed the problem of prescribedtime stability of linear systems with input delay. Some preliminaries on prescribed-time stabilization of linear systems without delay are discussed. The use of a generalized Vandermonde matrix (polynomial-based), as well as the Bell and Laguerre polynomials were instrumental for the analysis. Then, for the delayed case, the prescribed-time predictor feedback design is carried out based on the backstepping approach which makes use of time-varying kernels, for which, the bounded invertibility of the backstepping transformation is guaranteed.

Future work also includes prescribed-time stabilization of LTI systems with time-varying delays, distributed delays and unknown delays. The control design may use the ideas from delay-adaptive control combined with the methodology presented in this paper, i.e., still under backstepping approach with suitable transformations and choices of prescribed-time stable target systems. In addition, the problem of prescribedstabilization of reaction-diffusion systems with delayed boundary input is currently in progress which follows the essential ideas of this work. Some extensions can be done for prescribed-time output regulation and tracking for more general problems modeled by coupled ODE-PDEs.

\section{APPENDIX A}

\section{BELL, GENERALIZED LAGUERRE AND ELEMENTARY SYMMETRIC POLYNOMIALS}

\section{A. Complete and incomplete exponential Bell polynomials}

The exponential (partial) Bell polynomials are defined by the series expansion ([10]):

$$
\sum_{n=k}^{\infty} B_{n, k}\left(x_{1}, \ldots, x_{n-k+1}\right) \frac{t^{n}}{n !}=\frac{1}{k !}\left(\sum_{m=1}^{\infty} x_{m} \frac{t^{m}}{m !}\right)^{k} .
$$

The exponential (complete) Bell polynomials are defined by, $B_{0}=1$ and for $n \geq 0$,

$$
B_{n}\left(x_{1}, x_{2}, \ldots, x_{n}\right)=\sum_{k=1}^{n} B_{n, k}\left(x_{1}, x_{2}, \ldots, x_{n-k+1}\right) .
$$

Let us point out some relevant properties as follows:

Property 1 (Homogeneity): The following identity holds for $n \geq k$ and $a, b \in \mathbb{R}$.

$$
\begin{array}{r}
B_{n, k}\left(a b x_{1}, a b^{2} x_{2}, \ldots, a b^{n-k+1} x_{n-k+1}\right) \\
=a^{k} b^{n} B_{n, k}\left(x_{1}, x_{2}, \ldots, x_{n-k+1}\right)
\end{array}
$$

Property 2: Relation to the Lah numbers [10, Section 3.3]:

$$
B_{n, k}(1 !, \ldots,(n-k+1) !)=\left(\begin{array}{c}
n-1 \\
k-1
\end{array}\right) \frac{n !}{k !}=\operatorname{Lah}(n, k) \text {. }
$$

Property 3: Recurrence relation for the complete Bell polynomials [4] p. 270]:

$$
B_{n+1}\left(x_{1}, \ldots, x_{n+1}\right)=\sum_{k=0}^{n}\left(\begin{array}{l}
n \\
k
\end{array}\right) x_{n-k} B_{k}\left(x_{1}, \ldots, x_{k}\right) .
$$

\section{B. Generalized Laguerre polynomials}

Let us in addition introduce the the Kummer confluent hypergeometric function ${ }_{1} F_{1}(a ; b ; p)$.

$$
{ }_{1} F_{1}(a ; b ; s)=\sum_{k=0}^{\infty} \frac{(a)_{k}}{(b)_{k}} \frac{s^{k}}{k !}
$$

The Kummer function has the following property:

Property 4:

$$
\exp (s){ }_{1} F_{1}(a ; b ;-s)={ }_{1} F_{1}(b-a ; b ; s)
$$

A relation between the polynomials involving the Lah numbers (see Property 2) and the Kummer function is as follows:

Property 5:

$$
\sum_{k=1}^{n}\left(\begin{array}{l}
n-1 \\
k-1
\end{array}\right) \frac{n !}{k !} s^{k}=n ! s \exp (-s){ }_{1} F_{1}(n+1 ; 2 ; s)
$$

The generalized Laguerre polynomials are defined as follows ([39, Chapter 5]):

$$
L_{n}^{(\alpha)}(s)=\sum_{l=0}^{n}\left(\begin{array}{c}
n+\alpha \\
n-l
\end{array}\right) \frac{(-1)^{l}}{l !}(s)^{l}
$$

which can also be expressed in terms of 977:

$$
L_{k}^{(\alpha)}(s)=\left(\begin{array}{c}
k+\alpha \\
k
\end{array}\right){ }_{1} F_{1}(-k ; \alpha+1 ; s)
$$




\section{Elementary symmetric polynomials}

The elementary symmetric polynomials are defined as follows:

$$
\begin{aligned}
& \sigma_{0}\left(s_{1}, \ldots, s_{n}\right)=1, \\
& \sigma_{k}\left(s_{1}, \ldots, s_{n}\right)=\sum_{1 \leq i_{1} \leq i_{2} \leq \ldots i_{k} \leq n} s_{i_{1}} s_{i_{2}} \ldots s_{i_{k}}, \\
& \sigma_{n}\left(s_{1}, \ldots, s_{n}\right)=\prod_{i=1}^{n} s_{i},
\end{aligned}
$$

and $\sigma_{k}\left(s_{1}, \ldots, s_{n}\right)=0$, for $k>n$.

\section{APPENDIX B}

ProOF OF PROPOSITION2

Proof: We rewrite (10) as follows:

$$
\begin{aligned}
& \left(\delta^{n}\left(-r_{i} c\right)\right)(t)=B_{n+1}\left(-r_{i} c(t), \ldots,-r_{i} c^{(n)}(t)\right) \\
& =-r_{i} \sum_{k=0}^{n}\left(\begin{array}{l}
n \\
k
\end{array}\right) c^{(n-k)}(t) B_{k}\left(-r_{i} c(t), \ldots,-r_{i} c^{(k-1)}(t)\right) .
\end{aligned}
$$

By the definition of the complete Bell polynomials, we have

$$
\begin{aligned}
& \left(\delta^{n}\left(-r_{i} c\right)\right)(t)=B_{n+1}\left(-r_{i} c(t), \ldots,-r_{i} c^{(n)}(t)\right) \\
& =-r_{i} \sum_{k=0}^{n}\left(\begin{array}{l}
n \\
k
\end{array}\right) c^{(n-k)}(t) \sum_{j=0}^{k} B_{k, j}\left(-r_{i} c(t), \ldots,-r_{i} c^{(k-j)}(t)\right) .
\end{aligned}
$$

By (2) and computing iteratively its $(k-j)$-th derivative, (106) can be reformulated as follows:

$$
\begin{aligned}
& \left(\delta^{n}\left(-r_{i} c\right)\right)(t) \\
& =-r_{i} \sum_{k=0}^{n}\left(\begin{array}{l}
n \\
k
\end{array}\right) c^{(n-k)}(t) \\
& \quad \times \sum_{j=0}^{k} B_{k, j}\left(-r_{i} c(t),-r_{i} \dot{c}(t), \ldots,-r_{i} c^{(k-j)}(t)\right) \\
& =-r_{i} \sum_{k=0}^{n}\left(\begin{array}{l}
n \\
k
\end{array}\right) \frac{(2)_{n-k}}{\left(\bar{c}_{0} T\right)^{n-k}} c(t)(\sqrt{c(t)})^{n-k} \\
& \quad \times \sum_{j=0}^{k} B_{k, j}\left(-r_{i} c(t), \ldots,-r_{i} \frac{(2)_{k-j}}{\left(\bar{c}_{0} T\right)^{k-j}} c(t)(\sqrt{c(t)})^{k-j}\right) .
\end{aligned}
$$

From Property 1 we get

$$
\begin{aligned}
& \left(\delta^{n}\left(-r_{i} c\right)\right)(t)=-r_{i} \sum_{k=0}^{n}\left(\begin{array}{l}
n \\
k
\end{array}\right) \frac{(2)_{n-k}}{\left(\bar{c}_{0} T\right)^{n-k}} c(t)(\sqrt{c(t)})^{n-k} \\
& \quad \times \sum_{j=0}^{k} \frac{\left(-r_{i}\right)^{j}}{\left(\bar{c}_{0} T\right)^{k-j}} c^{j}(t)(\sqrt{c(t)})^{k-j} \times B_{k, j}\left((2)_{0}, \ldots,(2)_{k-j}\right),
\end{aligned}
$$

which can be simplified as follows:

$$
\begin{gathered}
\left(\delta^{n}\left(-r_{i} c\right)\right)(t)=-r_{i} \sum_{k=0}^{n}\left(\begin{array}{l}
n \\
k
\end{array}\right) \frac{(2)_{n-k}}{\left(\bar{c}_{0} T\right)^{n}} c(t)(\sqrt{c(t)})^{n} \\
\times \sum_{j=0}^{k}\left(-r_{i} \bar{c}_{0} T\right)^{j}(\sqrt{c(t)})^{j} B_{k, j}\left((2)_{0}, \ldots,(2)_{k-j}\right) .
\end{gathered}
$$

Notice that $B_{k, j}\left((2)_{0},(2)_{1},(2)_{2}, \ldots,(2)_{k-j}\right)=$ $B_{k, j}(1,2 !, 3 !, \ldots,(k-j+1) !)$. Therefore, applying Property 2 we have:

$$
\begin{gathered}
\left(\delta^{n}\left(-r_{i} c\right)\right)(t)=\frac{-r_{i} c(t)(\sqrt{c(t)})^{n}}{\left(\bar{c}_{0} T\right)^{n}} \sum_{k=0}^{n}\left(\begin{array}{l}
n \\
k
\end{array}\right)(2)_{n-k} \\
\times \sum_{j=0}^{k}\left(\begin{array}{l}
k-1 \\
j-1
\end{array}\right) \frac{k !}{j !}\left(-r_{i} \bar{c}_{0} T \sqrt{c(t)}\right)^{j} .
\end{gathered}
$$

Then, from Properties 4 and 5, it holds that

$$
\begin{aligned}
\left(\delta^{n}\left(-r_{i} c\right)\right)(t) & =\frac{-r_{i} c(t)(\sqrt{c(t)})^{n}}{\left(\bar{c}_{0} T\right)^{n}} \\
\times\left(\left(\begin{array}{l}
n \\
0
\end{array}\right)(2)_{n}\right. & +\sum_{k=1}^{n}\left(\begin{array}{l}
n \\
k
\end{array}\right)(2)_{n-k} k !\left(-r_{i} \bar{c}_{0} T \sqrt{c(t)}\right) \\
& \left.\times{ }_{1} F_{1}\left(1-k ; 2 ;\left(r_{i} \bar{c}_{0} T\right) \sqrt{c(t)}\right)\right) .
\end{aligned}
$$

After some simplifications and using (101), we obtain:

$$
\begin{aligned}
& \left(\delta^{n}\left(-r_{i} c\right)\right)(t)=\frac{-r_{i} c(t)(\sqrt{c(t)})^{n}}{\left(\bar{c}_{0} T\right)^{n}} n ! \\
& \times\left((n+1)+\left(\left(-r_{i} \bar{c}_{0} T \sqrt{c(t)}\right)\right.\right. \\
& \left.\quad \times \sum_{k=1}^{n} \frac{(n+1-k)}{k} L_{k-1}^{(1)}\left(r_{i} \bar{c}_{0} T \sqrt{c(t)}\right)\right) .
\end{aligned}
$$

For the next developments we need the following intermediate result:

$$
\sum_{j=1}^{n} \frac{L_{j-1}^{(1)}}{j}\left(r_{i} \bar{c}_{0} T \sqrt{c(t)}\right)=\frac{1}{r_{i} \bar{c}_{0} T \sqrt{c(t)}}\left(1-L_{n}^{(0)}\right)\left(r_{i} \bar{c}_{0} T \sqrt{c(t)}\right),
$$

along with the following well-known recurrence relations of the generalized Laguerre polynomials:

$$
\begin{aligned}
& \left(r_{i} \bar{c}_{0} T \sqrt{c(t)}\right) \sum_{k=0}^{n} L_{k}^{(\alpha)}\left(r_{i} \bar{c}_{0} T \sqrt{c(t)}\right)= \\
& (n+\alpha+1) L_{n}^{(\alpha)}\left(r_{i} \bar{c}_{0} T \sqrt{c(t)}\right)-(n+1) L_{n+1}^{(\alpha)}\left(r_{i} \bar{c}_{0} T \sqrt{c(t)}\right),
\end{aligned}
$$

and

$$
\begin{aligned}
& L_{n}^{(\alpha)}\left(r_{i} \bar{c}_{0} T \sqrt{c(t)}\right)=L_{n}^{(\alpha+1)}\left(r_{i} \bar{c}_{0} T \sqrt{c(t)}\right) \\
& -L_{n-1}^{(\alpha+1)}\left(r_{i} \bar{c}_{0} T \sqrt{c(t)}\right) .
\end{aligned}
$$

Hence, from (107) combined with (108) yields

$$
\begin{aligned}
& \left(\delta^{n}\left(-r_{i} c\right)\right)(t)= \\
& \frac{-r_{i} c(t)(\sqrt{c(t)})^{n}}{\left(\bar{c}_{0} T\right)^{n}} n !\left((n+1) L_{n}^{(0)}\left(r_{i} \bar{c}_{0} T \sqrt{c(t)}\right)\right. \\
& \left.-\left(-r_{i} \bar{c}_{0} T \sqrt{c(t)}\right) \sum_{k=1}^{n} L_{k-1}^{(1)}\left(r_{i} \bar{c}_{0} T \sqrt{c(t)}\right)\right) .
\end{aligned}
$$


Shifting the index in the above sum, we have

$$
\begin{aligned}
& \left(\delta^{n}\left(-r_{i} c\right)\right)(t)= \\
& \frac{-r_{i} c(t)(\sqrt{c(t)})^{n}}{\left(\bar{c}_{0} T\right)^{n}} n !\left((n+1) L_{n}^{(0)}\left(r_{i} \bar{c}_{0} T \sqrt{c(t)}\right)\right. \\
& \left.-\left(-r_{i} \bar{c}_{0} T \sqrt{c(t)}\right) \sum_{l=0}^{n-1} L_{l}^{(1)}\left(r_{i} \bar{c}_{0} T \sqrt{c(t)}\right)\right) .
\end{aligned}
$$

Using the first recurrence relation 109, we have

$$
\begin{aligned}
& \left(\delta^{n}\left(-r_{i} c\right)\right)(t)= \\
& \frac{-r_{i} c(t)(\sqrt{c(t)})^{n}}{\left(\bar{c}_{0} T\right)^{n}} n !\left((n+1) L_{n}^{(0)}\left(r_{i} \bar{c}_{0} T \sqrt{c(t)}\right)\right. \\
& \left.+(n+1) L_{n-1}^{(1)}\left(r_{i} \bar{c}_{0} T \sqrt{c(t)}\right)-n L_{n}^{(1)}\left(r_{i} \bar{c}_{0} T \sqrt{c(t)}\right)\right) .
\end{aligned}
$$

Applying the second recurrence relation (110), we finally obtain (11), that is 5 :

$\left(\delta^{n}\left(-r_{i} c\right)\right)(t)=\frac{-r_{i} c(t)(\sqrt{c(t)})^{n}}{\left(\bar{c}_{0} T\right)^{n}} n ! L_{n}^{(1)}\left(r_{i} \bar{c}_{0} T \sqrt{c(t)}\right)$.

This concludes the proof.

\section{APPENDIX C \\ PROOF OF PROPOSITION 3}

Proof: From (4), (7)-(9), the following equation (PDcharacteristic equation [24]) holds for all $i=1 . ., n$ :

$$
\begin{aligned}
& \left(\delta^{n-1}\left(-r_{i} c\right)\right)(t)+\sum_{j=2}^{n-1} p_{j}(t)\left(\delta^{j-1}\left(-r_{i} c\right)\right)(t)+p_{1}(t)\left(-r_{i} c(t)\right) \\
& \quad+p_{0}(t)=0 .
\end{aligned}
$$

In order to recover, $p_{0}, p_{j}(13)$-(14)), we will rewrite the problem as a linear system and proceed to solve it via inversion of the generalized Vandermonde matrix. From (113), we rewrite the problem to be solved in a more compact form

$$
V^{\top}(t) p(t)=Y(t)
$$

where $Y=\left(-\left(\delta^{n-1}\left(-r_{1} c\right)\right)(t), \ldots,-\left(\delta^{n-1}\left(-r_{n} c\right)\right)(t)\right)^{\top}$ and $V$ is given by (8) with $\left(\delta^{n}\left(-r_{i} c\right)\right)(t)=$ $\frac{-r_{i} c(t)(\sqrt{c(t)})^{n}}{\left(\bar{c}_{0} T\right)^{n}} n ! L_{n}^{(1)}\left(r_{i} \bar{c}_{0} T \sqrt{c(t)}\right)$ by virtue of Proposition 2 In addition, we recall the three-term recurrence relation of the generalized Laguerre polynomials,

$$
\begin{aligned}
& L_{0}^{(1)}\left(r_{i} \bar{c}_{0} T \sqrt{c(t)}\right)=1, \\
& L_{1}^{(1)}\left(r_{i} \bar{c}_{0} T \sqrt{c(t)}\right)=-r_{i} \bar{c}_{0} T \sqrt{c(t)}+2, \\
& n L_{n}^{(1)}\left(r_{i} \bar{c}_{0} T \sqrt{c(t)}\right)= \\
& \left(-r_{i} \bar{c}_{0} T \sqrt{c(t)}+2 n\right) L_{n-1}^{(1)}\left(r_{i} \bar{c}_{0} T \sqrt{c(t)}\right) \\
& \quad-(n) L_{n-2}^{(1)}\left(r_{i} \bar{c}_{0} T \sqrt{c(t)}\right), \quad n \geq 2,
\end{aligned}
$$

\footnotetext{
${ }^{5}$ Surprisingly, 11 turns out to be similar as the function $G^{(n)}(t)$ appearing in [16 Section 2.3] for prescribed-time stabilization of reaction-diffusion PDEs.
}

which allows to derive the following three-term-like recurrence relation:

$$
\begin{gathered}
\left(\delta^{n}\left(-r_{i} c\right)\right)(t)=-\frac{\sqrt{c(t)}}{\bar{c}_{0} T}\left(r_{i} \sqrt{c(t)}-2 n\right)\left(\delta^{n-1}\left(-r_{i} c\right)\right)(t) \\
-\frac{c(t)}{\left(\bar{c}_{0} T\right)^{2}} n(n-1)\left(\delta^{n-2}\left(-r_{i} c\right)\right)(t) .
\end{gathered}
$$

The generalized Vandermonde matrix (8) is a polynomialbased matrix that can be constructed using the classical Vandermonde matrix which is given as follows:

$$
V_{c}(t)=\left(\begin{array}{ccc}
1 & \cdots & 1 \\
-r_{1} c(t) & \cdots & -r_{n} c(t) \\
\vdots & \ddots & \vdots \\
\left(-r_{1} c(t)\right)^{n-1} & \cdots & \left(-r_{n} c(t)\right)^{n-1}
\end{array}\right) .
$$

Therefore, inspired by [5, Section 3], we observe that for $i=1, \ldots, n$, each column of the Vandermonde matrix $V$ can be recovered as follows:

$$
S_{\text {Lah }}(t)\left(\begin{array}{c}
1 \\
-r_{i} c(t) \\
\left(-r_{i} c(t)\right)^{2} \\
\vdots \\
\left(-r_{i} c(t)\right)^{n-1}
\end{array}\right)=\left(\begin{array}{c}
1 \\
\left(-r_{i} c(t)\right) \\
\left(\delta\left(-r_{i} c\right)\right)(t) \\
\vdots \\
\left(\delta^{n-2}\left(-r_{i} c\right)\right)(t)
\end{array}\right)
$$

provided that there exists a suitable lower triangular matrix (that we call in this framework $S_{\text {Lah }}(t)$ ) which captures the recurrence relation (116). Indeed, $S_{\text {Lah }}$ has the following structure:

$$
\begin{aligned}
& S_{\text {Lah }}(t)= \\
& \left(\begin{array}{cccccc}
1 & 0 & 0 & 0 & \cdots & 0 \\
0 & 1 & 0 & 0 & \cdots & 0 \\
0 & 2 \frac{\sqrt{c(t)}}{\bar{c}_{0} T} & 1 & 0 & \cdots & 0 \\
0 & 6 \frac{c(t)}{\left(\bar{c}_{0} T\right)^{2}} & 6 \frac{\sqrt{c(t)}}{\bar{c}_{0} T} & 1 & \ddots & \vdots \\
\vdots & \vdots & \vdots & \ddots & \ddots & \vdots \\
0 & (n-1) ! \frac{(\sqrt{c(t)})^{n-2}}{\left(\bar{c}_{0} T\right)^{n-2}} & \frac{(n-2)(n-1) !}{2} \frac{(\sqrt{c(t)})^{n-3}}{\left(\bar{c}_{0} T\right)^{n-3}} & \cdots & & 1
\end{array}\right) .
\end{aligned}
$$

Thus,

$$
S_{\mathrm{Lah}}(t) V_{c}(t)=V(t) \text {. }
$$

It is interesting to observe that the generalized Laguerre polynomials appearing in the Vandermonde matrix (8) have a close connection with the Lah numbers. Indeed, when looking at the rows of (119) yields the coefficients of the polynomial appearing in $V$. Inversely, when looking at the columns of (119) one realizes the Lah numbers. The entries of $S_{\text {Lah }}$ then involves the Lah numbers (suggesting the adopted name for such a matrix). More precisely, the entries $\left(S_{\mathrm{Lah}}\right)_{i j}$ of (119) are given by the following relations:

$\left(S_{\text {Lah }}\right)_{i j}=\left\{\begin{array}{l}\operatorname{Lah}(i-1, j-1) \frac{(\sqrt{c(t)})^{i-j}}{\left(\bar{c}_{0} T\right)^{i-j}}, \quad 1 \leq j \leq i \leq n \\ 0, \quad \text { otherwise },\end{array}\right.$

where $\operatorname{Lah}(i-1, j-1)=\left(\begin{array}{l}i-2 \\ j-2\end{array}\right) \frac{(i-1) !}{(j-1) !}$ (recall Property (2)) along with $\operatorname{Lah}((i-1), 0)=0, \operatorname{Lah}((i-1),(j-1))=0$ for all $j \geq i$. Due to the inversion formula of the Lah numbers [10], 
it turns out to be straightforward to compute the inverse of $S_{\mathrm{Lah}}(t)$. More precisely, the entries of $S_{\mathrm{Lah}}^{-1}(t)$ are as follows:

$$
\left(S_{\mathrm{Lah}}^{-1}\right)_{i j}= \begin{cases}(-1)^{i-j} \operatorname{Lah}(i-1, j-1) \frac{(\sqrt{c(t)})^{i-j}}{\left(\bar{c}_{0} T\right)^{i-j}} \\ 0, & 1 \leq j \leq i \leq n\end{cases}
$$

On the other hand, for the computation of the inverse of classical Vandermonde $V_{c}$ some algorithms make use of interpolation based techniques e.g. the Lagrange interpolation method and involve the elementary symmetric polynomials. Having said that, we follow e.g. [32], [41], [22] to compute the entries of the inverse of $V_{c}$. We obtain,

$$
\left(V_{c}^{-1}\right)_{i j}=\frac{(-1)^{n+1} \sigma_{n-j}\left(r_{1}, \ldots, r_{i-1}, r_{i+1}, \ldots, r_{n}\right)}{(c(t))^{j-1} \prod_{\substack{k=1 \\ k \neq 1 \\ k \neq i}}\left(r_{i}-r_{k}\right)}
$$

for $i=1, \ldots, n ; j=1, \ldots, n$; where $\sigma_{n-j}\left(r_{1}, \ldots, r_{i-1}, r_{i+1}, \ldots, r_{n}\right)$ is the $(n-j)$-th elementary symmetric polynomial (102)-(104) with $r_{i}$ deleted. Therefore, having the inverse of $V_{c}$, we are in position to characterize the inverse of the Vandermonde matrix (8). Indeed, from 120) in conjunction with (122) and 123, we get

$$
V^{-1}(t)=V_{c}^{-1}(t) S_{\text {Lah }}^{-1}(t) .
$$

Now, in order to solve (114), we can first solve $\tilde{p}=V_{c}^{-\top} Y$. Then, by the definition of the generalized Laguerre polynomials (100), and using (123), it follows that the $i$-th component of the vector $\tilde{p}$ can be computed explicitly,

$$
\begin{aligned}
& \tilde{p}_{i}(t)=(-1)^{n+1}(n-1) ! \frac{c(t)(\sqrt{c(t)})^{n-1}}{\left(\bar{c}_{0} T\right)^{n-1}} \\
& \times \sum_{j=1}^{n} \sum_{l=1}^{n} \frac{r_{j} \sigma_{n-i}\left(r_{1}, \ldots, r_{j-1}, r_{j+1}, \ldots, r_{n}\right)}{(c(t))^{i-1} \prod_{\substack{k=1 \\
k \neq j \\
k \neq j}}^{k=n}\left(r_{i}-r_{k}\right)} \\
& \left(\begin{array}{c}
n \\
n-l
\end{array}\right) \frac{\left(-r_{j} \bar{c}_{0} T \sqrt{c(t)}\right)^{l-1}}{(l-1) !}, \quad i=1, \ldots, n .
\end{aligned}
$$

Reorganizing terms, we get from 125):

$$
\begin{aligned}
& \tilde{p}_{i}(t)=(-1)^{n+1}(n-1) ! \frac{c(t)(\sqrt{c(t)})^{n-1}}{\left(\bar{c}_{0} T\right)^{n-1}(c(t))^{i-1}} \\
& \times \sum_{l=1}^{n}\left(\begin{array}{c}
n \\
n-l
\end{array}\right) \frac{\left(-\bar{c}_{0} T \sqrt{c(t)}\right)^{l-1}}{(l-1) !} \\
& \times \sum_{j=1}^{n} \frac{r_{j}^{l} \sigma_{n-i}\left(r_{1}, \ldots, r_{j-1}, r_{j+1}, \ldots, r_{n}\right)}{\prod_{\substack{k=n \\
k \neq 1 \\
k \neq j}}\left(r_{j}-r_{k}\right)} .
\end{aligned}
$$

Applying the following recurrence relation of the elementary symmetric polynomials into the last term of (126)

$$
\begin{gathered}
\sigma_{n-i+1}\left(r_{1}, \ldots, r_{n}\right)=\sigma_{n-i+1}\left(r_{1}, \ldots, r_{j-1}, r_{j+1}, \ldots, r_{n}\right) \\
+r_{j} \sigma_{n-i}\left(r_{1}, \ldots, r_{j-1}, r_{j+1}, \ldots, r_{n}\right),
\end{gathered}
$$

we get

$$
\begin{aligned}
\tilde{p}_{i}(t) & =(-1)^{n+1}(n-1) ! \frac{c(t)(\sqrt{c(t)})^{n-1}}{\left(\bar{c}_{0} T\right)^{n-1}(c(t))^{i-1}} \\
\times & \sum_{l=1}^{n}\left(\begin{array}{c}
n \\
n-l
\end{array}\right) \frac{\left(-\bar{c}_{0} T \sqrt{c(t)}\right)^{l-1}}{(l-1) !} \\
\times & \left(\sigma_{n-i+1}\left(r_{1}, \ldots, r_{n}\right) \mathcal{E}(n, l)+(-1)^{i-n} \mathcal{F}(i, l)\right),
\end{aligned}
$$

where

$$
\begin{aligned}
\mathcal{E}(n, l) & =\sum_{j=1}^{n} \frac{\left(r_{j}\right)^{l-1}}{\prod_{\substack{k=n \\
k \neq 1 \\
k \neq j}}\left(r_{j}-r_{k}\right)}, \\
\mathcal{F}(i, l) & =\sum_{j=1}^{n} \frac{(-1)^{n-i+1} \sigma_{n-i+1}\left(r_{1}, \ldots, r_{j-1}, r_{j+1}, \ldots, r_{n}\right)\left(r_{j}\right)^{l-1}}{\prod_{\substack{k=n \\
k=1 \\
k \neq j}}^{k=n}\left(r_{j}-r_{k}\right)} .
\end{aligned}
$$

We use then the orthogonality property related to the elementary symmetric polynomials and the invertibility of the classical Vandermonde matrix (see [41, Section 3]). Therefore, the following holds true:

$$
\mathcal{E}(n, l)=\delta_{n, l},
$$

where $\delta_{n, l}$ is the Kronecker delta function. Moreover,

$$
\mathcal{F}(i, l)=\delta_{i, l+1} \text {. }
$$

Thus, using 129) and (130), we can greatly simplify 128 and compute explicitly the $i$-th component of $\tilde{p}$ as follows:

$$
\tilde{p}_{1}(t)=\sigma_{n}\left(r_{1}, \ldots, r_{n}\right) c^{n}(t)
$$

and for $i=2, \ldots, n$, we get:

$$
\begin{aligned}
\tilde{p}_{i}(t)= & c^{n-i+1}(t) \sigma_{n-i+1}\left(r_{1}, \ldots, r_{n}\right) \\
& -\left(\begin{array}{c}
n \\
n-i+1
\end{array}\right) \frac{(n-1) !}{(i-2) !} \frac{(\sqrt{c(t)})^{n-i+1}}{\left(\bar{c}_{0} T\right)^{n-i+1}} .
\end{aligned}
$$

Hence, we can finally characterize $p$. It suffices to directly compute $p(t)=S_{\mathrm{Lah}}^{-\top}(t) \tilde{p}(t)$. Taking advantage of the upper triangular structure of $S_{\mathrm{Lah}}^{-\top}(t)$ (122) and from (131)-(132) we have that

$$
\begin{aligned}
& S_{\mathrm{Lah}}^{-\top}(t) \tilde{p}(t)=\sum_{j=i}^{n}(-1)^{j-i} \operatorname{Lah}(j-1, i-1) \\
& \times \frac{(\sqrt{c(t)})^{2 n-i-j+2}}{\left(\bar{c}_{0} T\right)^{j-i}} \sigma_{n-j+1}\left(r_{1}, \ldots, r_{n}\right) \\
& \quad+(-1)^{n-i+1}\left(\begin{array}{c}
n-1 \\
i-2
\end{array}\right) \frac{n !}{(i-1) !} \frac{(\sqrt{c(t)})^{n-i+1}}{\left(\bar{c}_{0} T\right)^{n-i+1}},
\end{aligned}
$$

where where $\operatorname{Lah}(j-1, i-1)=\left(\begin{array}{c}j-2 \\ i-2\end{array}\right) \frac{(j-1) !}{(i-1) !}$. Notice that 133 is equivalent to the following formula:

$$
\begin{aligned}
S_{\text {Lah }}^{-\top}(t) & \tilde{p}(t)=\sum_{j=i}^{n+1}(-1)^{j-i} \operatorname{Lah}(j-1, i-1) \\
& \times \frac{(\sqrt{c(t)})^{2 n-i-j+2}}{\left(\bar{c}_{0} T\right)^{j-i}} \sigma_{n-j+1}\left(r_{1}, \ldots, r_{n}\right) .
\end{aligned}
$$

Shifting the index, we get

$$
\begin{array}{r}
S_{\text {Lah }}^{-\top}(t) \tilde{p}(t)=\sum_{j=i-1}^{n}(-1)^{j-i+1} \operatorname{Lah}(j, i-1) \\
\times \frac{(\sqrt{c(t)})^{2 n-i-j+1}}{\left(\bar{c}_{0} T\right)^{j-i+1}} \sigma_{n-j}\left(r_{1}, \ldots, r_{n}\right) .
\end{array}
$$

Setting $k=j$ and reorganizing terms we have

$$
\begin{aligned}
& S_{\text {Lah }}^{-\top}(t) \tilde{p}(t)=\frac{(\sqrt{c(t)})^{n-i+1}}{\left(\bar{c}_{0} T\right)^{n-i+1}} \sum_{k=i-1}^{n}\left((-1)^{k-i+1}\right. \\
& \left.\times \operatorname{Lah}(k, i-1) \sigma_{n-k}\left(r_{1}, \ldots, r_{n}\right)\left(\bar{c}_{0} T \sqrt{c(t)}\right)^{n-k}\right) .
\end{aligned}
$$


Setting $j=i-1$, from (135), we finally obtain

$$
p_{0}(t)=\sigma_{n}\left(r_{1}, . ., r_{n}\right) c^{n}(t),
$$

and for $j=1, \ldots, n-1$,

$$
\begin{aligned}
p_{j}(t) & =\frac{(\sqrt{c(t)})^{n-j}}{\left(\bar{c}_{0} T\right)^{n-j}} \sum_{k=j}^{n}(-1)^{k-j}(\operatorname{Lah}(k, j) \\
& \left.\times \sigma_{n-k}\left(r_{1}, \ldots, r_{n}\right)\left(\bar{c}_{0} T \sqrt{c(t)}\right)^{n-k}\right) .
\end{aligned}
$$

This concludes the proof.

\section{APPENDIX D \\ PROOF OF LEMMA 4}

Proof: Let us first find an upper bound for $N_{\bar{\gamma}}(t-h)$ which is given by (83). It requires to exploits the structure of the Vandermonde matrix and its inverse (see (11) along with (120)-(124)). Therefore, an estimate for the scalar product has the following form:

$$
\bar{\gamma}^{\top}(t-h, x) \bar{\gamma}(t-h, x) \leq \mathcal{Q}(\sqrt{c(t-h)}) \mathcal{S}(t-h, x),
$$

with

$$
\begin{aligned}
\mathcal{S}(t-h, x) & =\sum_{\substack{1 \leq i \leq j \leq n \\
\\
\times \mathfrak{e}_{\left(r_{i}+r_{j}\right)}(t-h) \mathfrak{e}_{-\left(r_{i}+r_{j}\right)}(t-h+x)}}\left(g_{i, j}^{0}+g_{i, j}^{1}(\sqrt{c(t-h+x)})\right)
\end{aligned}
$$

where $\mathcal{Q}(\sqrt{c(t-h)})$ is a rational polynomial function (whose coefficients depend on $r_{i}, a_{i-1}, \bar{c}_{0}$, and $T$ ); and $g_{i, j}^{1}$ are polynomials of $\sqrt{c(t-h+x)}$ (whose coefficients depend on $r_{i}, a_{i-1}, \bar{c}_{0}$ and $\left.T\right)$. In addition, $g_{i, j}^{0}$ are positive scalars depending on $r_{i}, a_{i-1}, i=1, \ldots, n$ as well as on $\bar{c}_{0}$ and $T$.

Therefore, integrating (136) and rearranging terms, we obtain,

$$
\int_{0}^{h}\|\bar{\gamma}(t-h, x)\|^{2} d x \leq \mathcal{Q}(\sqrt{c(t-h)}) \mathcal{I}(t-h),
$$

with $\mathcal{I}(t-h)=\sum_{1 \leq i \leq j \leq n} \mathfrak{e}_{\left(r_{i}+r_{j}\right)}(t-h)\left(\mathcal{G}_{i, j}^{0}+\mathcal{G}_{i, j}^{1}\right)$, where

$\mathcal{G}_{i, j}^{0}=g_{i, j}^{0} \int_{0}^{h} \mathfrak{e}_{-\left(r_{i}+r_{j}\right)}(t-h+x) d x$,

$$
\mathcal{G}_{i, j}^{1}=\int_{0}^{h} g_{i, j}^{1}(\sqrt{c(t-h+x)}) \mathfrak{e}_{-\left(r_{i}+r_{j}\right)}(t-h+x) d x .
$$

Let us first analyze $\mathcal{G}_{i, j}^{1}$. Since $g_{i, j}^{1}(\sqrt{c(t-h+x)})$ is a polynomial in $\sqrt{c(t-h+x)}$, 139) can be explicitly computed which in turn allows to obtain an upper bound as follows:

$$
\begin{aligned}
& \mathcal{G}_{i, j}^{1} \leq \mathcal{G}_{i, j}^{11}, \\
& \mathcal{G}_{i, j}^{11}(t-h)=g_{i, j}^{11}(\sqrt{c(t-h)}) \mathfrak{e}_{-\left(r_{i}+r_{j}\right)}(t-h),
\end{aligned}
$$

with $g_{i, j}^{11}(\sqrt{c(t-h)})$ a polynomial function of $\sqrt{c(t-h)}$. On the other hand, $\mathcal{G}_{i, j}^{0}$ in (138) is much more involved. In order to get an upper bound of it, we are going to use the socalled generalized Exponential integral function and exploit its properties. Let us first rewrite (138) as follows:

$$
\mathcal{G}_{i, j}^{0}(t)=g_{i, j}^{0} \frac{r_{i}+r_{j}}{2}\left(\bar{c}_{0} T\right)^{2} \int_{\left(r_{i}+r_{j}\right) \bar{c}_{0} T \sqrt{c(t-h)}}^{\left(r_{i}+r_{j}\right) \bar{c}_{0} T \sqrt{c(t)}} \frac{\exp (-s)}{s^{2}} d s,
$$

where the change of variable $s=\left(r_{i}+r_{j}\right) \bar{c}_{0} T \sqrt{c(t-h+x)}$ has been used. The form of (140) allows to use the following generalized exponential integral:

$$
E_{n}(v)=v^{n-1} \int_{v}^{\infty} \frac{\exp (-s)}{s^{n}} d s, \quad v>0, n \in \mathbb{N} .
$$

Indeed, by virtue of the definition of generalized exponential integral (141), we split the integral $\int_{\left(r_{i}+r_{j}\right) \bar{c}_{0} T \sqrt{c(t-h)}}^{\left(r_{i}+r_{j}\right) \bar{c} T \sqrt{c()}} \frac{\exp (-s)}{s^{2}} d s$ in (140) as follows: $\int_{\left(r_{i}+r_{j}\right) \bar{c}_{0} T \sqrt{c(t-h)}}^{\infty} \frac{\exp (-s)}{s^{2}} d s-\int_{\left(r_{i}+r_{j}\right) \bar{c}_{0} T \sqrt{c(t)}}^{\infty} \frac{\exp (-s)}{s^{2}} d s$ so that

$$
\begin{array}{r}
\int_{\left(r_{i}+r_{j}\right) \bar{c}_{0} T \sqrt{c(t-h)}}^{\infty} \frac{\exp (-s)}{s^{2}} d s=\frac{E_{2}\left(\left(r_{i}+r_{j}\right) \bar{c}_{0} T \sqrt{c(t-h)}\right)}{\left(r_{i}+r_{j}\right) \bar{c}_{0} T \sqrt{c(t-h)}}, \\
\int_{\left(r_{i}+r_{j}\right) \bar{c}_{0} T \sqrt{c(t)}}^{\infty} \frac{\exp (-s)}{s^{2}} d s=\frac{E_{2}\left(\left(r_{i}+r_{j}\right) \bar{c}_{0} T \sqrt{c(t)}\right)}{\left(r_{i}+r_{j}\right) \bar{c}_{0} T \sqrt{c(t)}} .
\end{array}
$$

Moreover, the following inequalities hold [9, Section 2]:

$$
0<\frac{\exp (-v)}{2+v} \leq E_{2}(v) \leq \frac{\exp (-v)}{1+v}, \quad v>0 .
$$

Hence, by re-arranging terms and using (142), we obtain that an upper bound for $\mathcal{G}_{i, j}^{0}(t)$ is given as follows:

$$
\begin{aligned}
\mathcal{G}_{i, j}^{0} & \leq \mathcal{G}_{i, j}^{01}, \\
\mathcal{G}_{i, j}^{01}(t-h) & =\frac{\left(r_{i}+r_{j}\right)\left(\bar{c}_{0} T\right)^{2} g_{i, j}^{0}}{2 \mathfrak{s}_{\left(r_{i}+r_{j}\right)}^{-1}(t-h)} \frac{\mathfrak{e}_{-\left(r_{i}+r_{j}\right)}(t-h)}{1+\mathfrak{s}_{\left(r_{i}+r_{j}\right)}^{-1}(t-h)} .
\end{aligned}
$$

Therefore, by combining (144) and (140) we obtain from (137) the estimate for $N_{\bar{\gamma}}(t-h)$ as follows:

$$
\begin{array}{r}
N_{\bar{\gamma}}(t-h) \leq 2 \mathcal{Q}(\sqrt{c(t-h)}) \sum_{1 \leq i \leq j \leq n} \mathfrak{e}_{\left(r_{i}+r_{j}\right)}(t-h) \\
\times\left(\mathcal{G}_{i, j}^{01}(t-h)+\mathcal{G}_{i j}^{11}(t-h)\right) .
\end{array}
$$

Finally, by virtue of Lemma 3 in conjunction with (145), we get the following:

$$
\begin{aligned}
& N_{\bar{\gamma}}(t-h) P(\sqrt{c(t-h)}) \mathfrak{e}_{-r_{\text {min }}}(t-h) \leq 2 P(\sqrt{c(t-h)}) \\
& \mathcal{Q}(\sqrt{c(t-h)}) \mathfrak{e}_{-r_{\text {min }}}(t-h) \sum_{1 \leq i \leq j \leq n} \mathfrak{e}_{\left(r_{i}+r_{j}\right)}(t-h) \\
& \times\left(\mathcal{G}_{i, j}^{01}(t-h)+\mathcal{G}_{i j}^{11}(t-h)\right) .
\end{aligned}
$$

Notice that

$$
\mathfrak{e}_{\left(r_{i}+r_{j}\right)}(t-h) \mathcal{G}_{i, j}^{01}(t-h)=\vartheta \frac{\mathfrak{e}_{\left(r_{i}+r_{j}\right)}(t-h) \mathfrak{e}_{-\left(r_{i}+r_{j}\right)}(t-h)}{\mathfrak{s}_{\left(r_{i}+r_{j}\right)}^{-1}(t-h)\left(1+\mathfrak{s}_{\left(r_{i}+r_{j}\right)}^{-1}(t-h)\right)},
$$

where $\vartheta$ is a constant whose specific characterization is not needed in the remaining part of the analysis. Since $\mathfrak{e}_{-r}(s) \mathfrak{e}_{r}(s)=1$, we conclude that the terms $\mathfrak{e}_{\left(r_{i}+r_{j}\right)}(t-$ $h) \mathcal{G}_{i, j}^{01}(t-h)$ and $\mathfrak{e}_{\left(r_{i}+r_{j}\right)}(t-h) \mathcal{G}_{i, j}^{11}(t-h) \rightarrow 0$ as $t \rightarrow t_{0}+h+$ $T$. Therefore, from (146), $N_{\bar{\gamma}}(t-h) P(\sqrt{c(t-h)}) \mathfrak{e}_{-r_{\text {min }}}(t-$ $h) \rightarrow 0$ as $t \rightarrow t_{0}+h+T$. This concludes the proof.

\section{ACKNOWLEDGMENT}

The authors would like to thank Drew Steeves and Miroslav Krstic for the valuable comments on the preliminary version of this paper. 


\section{REFERENCES}

[1] Z. Artstein. Linear systems with delayed controls: A reduction. IEEE Transactions on Automatic Control, 27:869-879, 1982.

[2] J. Auriol and F. Di Meglio. Minimum time control of heterodirectional linear coupled hyperbolic PDEs. Automatica, 71:300-307, 2016.

[3] N. Bekiaris-Liberis and M. Krstic. Lyapunov stability of linear predictor feedback for distributed input delays. IEEE Transactions on Automatic Control, 56(3):655-660, 2011.

[4] E. T. Bell. Exponential polynomials. Annals of Mathematics, 35(2):258277, 1934

[5] T. Bella, Y. Eidelman, I. Gohberg, I. Koltracht, and V. Olshevsky. A Björck-Pereyra-type algorithm for Szegö - Vandermonde matrices based on properties of unitary hessenberg matrices. Linear Algebra and its Applications, 420(2):634 - 647, 2007.

[6] S.P. Bhat and D.S. Bernstein. Finite time stability of continuous autonomous systems. SIAM J. Control Optim., 38(3):751-766, 2000.

[7] D. Bresch-Pietri and M. Krstic. Delay-adaptive predictor feedback for systems with unknown long actuator delay. IEEE Transactions on Automatic Control, 55(9):2106-2112, 2010.

[8] D. Bresch-Pietri and M. Krstic. Delay-adaptive control for nonlinear systems. IEEE Transactions on Automatic Control, 59(5):1203-1218, 2014.

[9] C. Chiccoli, S. Lorenzutta, and G. Maino. Recent results for generalized exponential integrals. Computer \& Mathematics with Applications, 19(5):21-29, 1990.

[10] L. Comtet. Advanced Combinatorics. D. Reidel Publishing Co, 1974.

[11] J.-M. Coron, L. Hu, and G. Olive. Finite-time boundary stabilization of general linear hyperbolic balance laws via fredholm backstepping transformation. Automatica, 84:95-100, 2017.

[12] J.-M. Coron and H.-M. Nguyen. Null controllability and finite time stabilization for the heat equations with variable coefficients in space in one dimension via backstepping approach. Archive for Rational Mechanics and Analysis, 225(3):993-1023, 2017.

[13] J. Deutscher. Finite-time output regulation for linear $2 \times 2$ hyperbolic systems using backstepping. Automatica, 75:54 - 62, 2017.

[14] D. Efimov, P. Polyakov, E. Fridman, W. Perruquetti, and J.-P. Richard. Comments on finite-time stability of time-delay systems. Automatica, 50(7):1944 - 1947, 2014.

[15] N. Espitia and W. Perruquetti. Prescribed-time predictor control of LTI systems with input delay. In 59th IEEE Conference on Decision and Control (CDC), Jeju Island, Republic of Korea, 2020.

[16] N. Espitia, A. Polyakov, D. Efimov, and W. Perruquetti. Boundary time-varying feedbacks for fixed-time stabilization of constant-parameter reaction-diffusion systems. Automatica, 103:398 - 407, 2019.

[17] N. Espitia, A. Polyakov, D. Efimov, and W. Perruquetti. Some characterizations of boundary time-varying feedbacks for fixed-time stabilization of reaction-diffusion systems. IFAC-PapersOnLine, 52(2):162 - 167, 2019. 3rd IFAC Workshop on Control of Systems Governed by Partial Differential Equations CPDE 2019.

[18] V.T. Haimo. Finite time controllers. SIAM Journal of Control and Optimization, 24(4):760-770, 1986

[19] J. Holloway and M. Krstic. Prescribed-time observers for linear systems in observer canonical form. IEEE Transactions on Automatic Control, 64(9):3905-3912, 2019.

[20] J. Holloway and M. Krstic. Prescribed-time output feedback for linear systems in controllable canonical form. Automatica, 107:77-85, 2019.

[21] E. Jiménez-Rodríguez, AJ. Muñoz Vázquez, JD. Sánchez-Torres, M. Defoort, and AG. Loukianov. A Lyapunov-like characterization of predefined-time stability. IEEE Transactions on Automatic Control, pages 1-1, 2020.

[22] T. Kailath and V. Olshevsky. Displacement-structure approach to polynomial Vandermonde and related matrices. Linear Algebra and its Applications, 261(1):49 - 90, 1997.

[23] EW. Kamen. Fundamentals of Linear Time-Varying Systems, chapter 3, page 17. Taylor \& Francis Group.

[24] EW. Kamen. The poles and zeros of a linear time- varying system. Linear Algebra and its Applications, (98):263-289, 1988.

[25] I. Karafyllis. Finite-time global stabilization by means of time-varying distributed delay feedback. SIAM J. Control Optim., 45:320-342, 2006.

[26] P. Krishnamurthy, F. Khorrami, and M. Krstic. A dynamic high-gain design for prescribed-time regulation of nonlinear systems. Automatica, 115:108860, 2020.

[27] M. Krstic. Delay Compensation for Nonlinear, Adaptive, and PDE Systems. Birkhäuser, 2009.
[28] M. Krstic and A. Smyshlyaev. Backstepping boundary control for firstorder hyperbolic PDEs and application to systems with actuator and sensor delays. Systems \& Control Letters, 57(9):750-758, 2008.

[29] F. Lopez-Ramirez, A. Polyakov, D. Efimov, and W. Perruquetti. Finitetime and fixed-time observer design: Implicit lyapunov function approach. Automatica, 87:52 - 60, 2018

[30] F. Mazenc, M. Malisoff, and S.-I. Niculescu. Reduction model approach for linear time-varying systems with delays. IEEE Transactions on Automatic Control, 59(2068-2081), 2014.

[31] E. Moulay, M. Dambrine, N. Yeganefar, and W. Perruquetti. Finite-time stability and stabilization of time-delay systems. Systems \& Control Letters, 57:561-566, 2008.

[32] V.-E. Neagoe. Inversion of the Vandermonde matrix. IEEE Signal Processing Letters, 3(4):119-120, 1996.

[33] V. Perrollaz and L. Rosier. Finite-time stabilization of $2 \times 2$ hyperbolic systems on tree-shaped networks. SIAM Journal on Control and Optimization, 52(1):143-163, 2014.

[34] A. Polyakov, J.-M. Coron, and L. Rosier. On Homogeneous Finite-Time Control For Evolution Equation in Hilbert Space. IEEE Transactions on Automatic Control, 63(9):3143-3150, 2017.

[35] A. Polyakov, D. Efimov, and W. Perruquetti. Finite-time and Fixedtime Stabilization: Implicit Lyapunov Function Approach. Automatica, 51(1):332-340, 2015.

[36] L.F. Shampine. Two-step Lax-Friedrichs method. Applied Mathematics Letters, 18(10):1134-1136, 2005.

[37] Y.-D. Song, Y.-J. Wang, J.-C. Holloway, and M. Krstic. Time-varying feedback for regulation of normal-form nonlinear systems in prescribed finite time. Automatica, 83:243 - 251, 2017.

[38] D. Steeves, M. Krstic, and R. Vazquez. Prescribed-time H1-stabilization of reaction-diffusion equations by means of output feedback. In 2019 18th European Control Conference (ECC), pages 1932-1937, 2019.

[39] G. Szego. Orthogonal polynomials, volume 23. American Mathematical Society Providence, Rhode Island, 4th edition, 1975.

[40] D. Tran and T. Yucelen. Finite-time control of perturbed dynamical systems based on a generalized time transformation approach. Systems \& Control Letters, 136:104605, 2020.

[41] J. Traub. Associated polynomials and uniform methods for the solution of linear problems. SIAM Rev, 8(3):277-301, 1966.

[42] B. Zhou. Finite-time stability analysis and stabilization by bounded linear time-varying feedback. Automatica, 121, 2020.

[43] J.J. Zhu and C. D. Johnson. Unified canonical forms for linear timevarying dynamical systems under d-similarity transformations. ii. In The Twenty-First Southeastern Symposium on System Theory, pages 57-63, 1989.

[44] Z. Zou. Fixed-time stabilization of general linear systems with input delay. Journal of the Franklin Institute, 356(8):4467-4477, 2019. 